\title{
Outstanding visible photocatalytic activity of a new mixed
}

\section{Bismuth Titanatate material}

\author{
P. Zambrano ${ }^{1,2}$, M.J. Sayagués ${ }^{1}$, J.A. Navío ${ }^{1}$ and M.C. Hidalgo ${ }^{1 *}$ \\ ${ }^{1}$ Instituto de Ciencia de Materiales de Sevilla, CSIC-Universidad de Sevilla, Americo \\ Vespucio 49, 410092, Sevilla, Spain \\ ${ }^{2}$ Departamento Cristalografía, Mineralogía y Química Agrícola, Universidad de Sevilla, \\ C/Profesor García González s/n, 41012 Sevilla, Spain
}

\begin{abstract}
In this work, a new photocatalyst based on bismuth titanates with outstanding visible photocatalytic activity was prepared by a facile hydrothermal method. The synthesised material showed visible activity as high as UV activity of commercial $\mathrm{TiO}_{2}$ P25 under the same experimental conditions for phenol degradation. A wide characterisation of the photocatalyst was performed. The material was composed of three phases; majority of $\mathrm{Bi}_{20} \mathrm{TiO}_{32}$ closely interconnected to $\mathrm{Bi}_{4} \mathrm{Ti}_{3} \mathrm{O}_{12}$ and amorphous $\mathrm{TiO}_{2}$. The high visible activity showed by this material could be ascribed to a combination of several features; i.e. low band gap energy value $(2.1 \mathrm{eV})$, a structure allowing a good separation path for visible photogenerated electron-holes pairs and a relatively high surface area. This photocatalyst appeared as a promising material for solar and visible applications of photocatalysis.
\end{abstract}

Keywords: visible photocatalysis, $\mathrm{Bi}_{4} \mathrm{Ti}_{3} \mathrm{O}_{12}, \mathrm{Bi}_{20} \mathrm{TiO}_{32}$, phenol degradation

\footnotetext{
Corresponding author: Tel: +34 954489501

*E-mail: carmen.hidalgo@csic.es
} 


\section{Introduction}

Titanium dioxide $\left(\mathrm{TiO}_{2}\right)$ has been until now the most studied material used as photocatalyst, due to its good properties such as nontoxicity, chemical and photochemical stability and remarkable photoactivity under ultraviolet irradiation [12]. However, there are also some drawbacks of this material which hinder its wide use for many applications; mainly its high recombination rate of photogenerated electronholes which lower its photoefficiency and its inability to be active under visible light. Due to its large band-gap $\mathrm{TiO}_{2}$ can only absorb light in the ultraviolet region which limits its implementation for solar and indoor applications [3]. Thus, in the last years, a lot of effort in the photocatalysis research has been focused in the development and preparation of alternative materials to $\mathrm{TiO}_{2}$ for solar and visible photocatalytic applications [4-6]. In this sense the searching of new or modified materials with absorption in the visible range of the spectrum has been a deal in many studies with the objective of obtaining materials with also a lower recombination rate of photogenerated charges for getting higher efficiency in the photocatalytic processes [4-8].

In this context, the family of bismuth titanates has attracted the interest of researchers in the area due to promising positive results in visible photocatalysis. Bismuth titanates include several different phases such as $\mathrm{Bi}_{2} \mathrm{Ti}_{2} \mathrm{O}_{7}, \mathrm{Bi}_{2} \mathrm{Ti}_{4} \mathrm{O}_{11}, \mathrm{Bi}_{4} \mathrm{Ti}_{3} \mathrm{O}_{12}, \mathrm{Bi}_{12} \mathrm{TiO}_{20}$, $\mathrm{Bi}_{20} \mathrm{TiO}_{32}$, etc. [9]. Probably the most studied phase, for photocatalytic applications, is the $\mathrm{Bi}_{4} \mathrm{Ti}_{3} \mathrm{O}_{12}$, which has shown high or moderate photocatalytic activity under visible illumination in several studies [10-15]. Although the phase $\mathrm{Bi}_{20} \mathrm{TiO}_{32}$ has also appeared as photoactive, it has been much less studied due to its metastable character, which makes it difficult to obtain as single phase or by means of mild conditions preparation 
methods. The visible activity of $\mathrm{Bi}_{20} \mathrm{TiO}_{32}$ is ascribed to the hybridized valence band by Bi6s and $02 p$ orbitals which lead to a low band gap value [16]. This phase has been mainly obtained by synthetic procedures involving severe conditions as quenching methods at high temperatures [17-20]. This kind of preparation methods usually gives low surface areas and highly irregular materials, which in principle are negative features for catalysts. Therefore, when these materials are expected to be used as photocatalysts it would be worth to study alternative synthetic methods to get this kind of materials using mild preparation conditions [20].

In this paper, materials based in $\mathrm{Bi}-\mathrm{Ti}-\mathrm{O}$ have been prepared with an extraordinary activity in the visible, evaluated in the phenol photodegradation reaction. This model compound was chosen due to its wide utilisation in environmental photocatalytic studies [21-22]. Additionally, as visible activity was also evaluated, it appears as more appropriate substrate than dies, which have been also often used but that could present photosensibilisation effects masking real visible activity [23].

A wide characterization has been performed in order to understand the good visible photocatalytic performance. As reference material $\mathrm{TiO}_{2}$ Degussa P25 was used and it was observed that the visible activity of the obtained Bi-Ti-O material was comparable to the activity of $\mathrm{TiO}_{2} \mathrm{P} 25$ under the ultraviolet illumination under the same experimental parameters. This, as far as we know, has not often been reported. The materials obtained are then very promising for any kind of solar application and/or indoors application, where visible light would be the source of illumination.

\section{Experimental}

\subsection{Catalysts preparation}


The Bi-Ti-O sample was prepared by mixing two solutions with the corresponding precursors and a further hydrothermal treatment.

First solution (solution A) was obtained by mixing Titanium tetraisopropoxide (TTiP Aldrich 97\%) in Isopropanol (Aldrich 99.9\%) and distilled water. This solution was kept under stirring at room temperature for one week to obtain a milky sol, as described in a previous paper [24].

Second solution (solution $\mathrm{B}$ ) was prepared by dissolving $\mathrm{Bi}\left(\mathrm{NO}_{3}\right)_{3} \cdot 5 \mathrm{H}_{2} \mathrm{O}$ (analytical grade from Aldrich) in glacial acetic acid (reactive grade from Aldrich) to obtain a $1 \mathrm{M}$ solution.

Then, solution B was slowly added to solution A and kept under stirring for one hour. Afterwards, triethylamine (TEA, Aldrich $>99.5 \%$ ) was added until reaching a $\mathrm{pH}$ value of 9. The resulting sol was transferred into a Teflon recipient inside of a stainless steel autoclave and hydrothermal treatment was performed at $140 \circ \mathrm{C}$ for 20 hours. A precipitate was obtained and was then filtered, repeatedly washed with distilled water and dried overnight at $120^{\circ} \mathrm{C}$.

The obtained powder was divided in two portions; one of them was calcined at $400^{\circ} \mathrm{C}$ for 2 hours. Hereafter, the non-calcined sample is named as BTO-Fresh, and the calcined one BTO-400.

On the other hand, commercial $\mathrm{TiO}_{2}$ Degussa P25 was employed "as received" without previous treatment.

\subsection{Characterisation of the catalysts}


Crystalline phase composition and degree of crystallinity of the samples were estimated by X-ray diffraction (XRD), on a Siemens D-501 diffractometer with Ni filter and graphite monochromator using $\mathrm{Cu}$ Ka radiation (0.15406 nm).

Global composition was determined by X-ray fluorescence spectrometry (XRF) in a Panalytical Axios sequential spectrophotometer with a rhodium tube as the source of radiation. XRF measurements were performed onto pressed pellets (sample included in $10 w t \%$ of wax).

BET surface area and porosity measurements were carried out by $\mathrm{N}_{2}$ adsorption at $77 \mathrm{~K}$ using a Micromeritics 2010 instrument.

The morphology and microcharacterization of the samples were analyzed using scanning (SEM) and transmission (TEM) electron microscopy techniques. The samples were dispersed in ethanol using an ultrasonicator and dropped on a carbon coated copper grid. For SEM observations, a Hitachi S 4800 field emission-SEM was used in secondary electron mode at an acceleration voltage of $5 \mathrm{kV}$ with an EDX detector for chemical analysis. The samples were sufficiently conductive, thus they were not coated with any conductive material in order to avoid charging artefacts.

For TEM studies, a FEI TECNAI G2 F30 S-twin high resolution transmission electron microscope (HRTEM) was used, with field emission gun (FEG) and scanningtransmission capabilities (STEM) operating at $300 \mathrm{kV}$ and $0.2 \mathrm{~nm}$ point resolution. The microscope was equipped with a high angle annular dark field (HAADF) detector with $0.16 \mathrm{~nm}$ point resolution from Fischione Instruments, and an INCA X-Max 80 silicon drift detector (SDD) for the energy dispersive X-ray analysis (EDX). The Gatan Digital Micrograph software (Gatan Inc.) was used to measure lattice spacing and to calculate the First Fourier Transform (FFT) and the Java version Electron Microscope Software 
(JEM) was used to analysed the different phases of the samples. The analysis of the HAAD-STEM images and the EDX spectra profile were done with the ES Vision software (FEI Company).

UV-vis spectra were recorded by using a Cary 100 (Varian) in the diffuse reflectance mode $(R)$ and transformed to a magnitude proportional to the extinction coefficient $(K)$ through the Kubelka-Munk function, $F(R \infty)$. Samples were mixed with $\mathrm{BaSO}_{4}$ that does not absorb in the UV-vis radiation range (white standard). Scans range was $240-800$ $\mathrm{nm}$.

\subsection{Photocatalytic activity}

The photocatalytic activity of the samples was evaluated in the phenol oxidation reaction using a batch reactor $(250 \mathrm{ml})$ and an Osram Ultra-Vitalux lamp $(300 \mathrm{~W})$ with sunlike radiation spectrum. A concentration of $1 \mathrm{~g} / \mathrm{L}$ of photocatalyst was used and the initial concentration of phenol was $50 \mathrm{ppm}$.

The intensity of the incident UVA light on the solution was measured with a PMA 2200 UVA photometer (Solar Light Co.) being ca. $90 \mathrm{~W} / \mathrm{m}^{2}$ (UVA PMA2110 sensor; spectral response $320-400 \mathrm{~nm}$ ). On the other hand, the intensity of light in the visible range measured in this case was $110 \mathrm{~W} / \mathrm{m}^{2}$ (Photopic PMA21300 sensor; spectral response 400-700 nm). The photocatalytic runs in the visible range were performed by using a polyester UV filter sheet (Edmund Optics) showing 99.9\% of absorbance below 390 $\mathrm{nm}$.

In the oxidation tests, an oxygen flow was employed what produces a homogenous suspension of the catalyst in the solution. Before each experiment, the catalysts were 
settled in suspension with the reagent mixture for $20 \mathrm{~min}$ to allow catalyst-phenol adsorption equilibration.

The evolution of the phenol concentration was followed through HPLC analysis (Agilent, 1200 Serie) using filtered aliquots ca. $2 \mathrm{ml}$ of the suspension (Millipore Millex25 $0.45 \mathrm{~mm}$ membrane filter).

Blank experiments were performed in the dark with catalyst as well as with illumination and no catalyst, and no changes in the initial concentration of phenol were observed in any of both cases.

\section{Results and discussion}

\subsection{Characterisation}

Figure 1 show XRD patterns of the fresh sample and after calcination treatment. As it can be seen, the sample is already crystalline before calcination and the only phase present can be identified as $\mathrm{Bi}_{4} \mathrm{Ti}_{3} \mathrm{O}_{12}$ (PDF-012-02-13). After calcination at 400 으 for 2h, two crystalline phases can be identified, i.e $\mathrm{Bi}_{20} \mathrm{TiO}_{32}$ (PDF-042-02-02) and $\mathrm{Bi}_{4} \mathrm{Ti}_{3} \mathrm{O}_{12}$; with estimated percentages of $77 \%$ and $23 \%$ respectively (see Table 1 ). For comparison XRD pattern of commercial $\mathrm{TiO}_{2}$ Degussa P25 is also shown, where anatase and rutile phases of titania can be seen in a ratio 80:20 respectively [25].

$\mathrm{Bi}_{4} \mathrm{Ti}_{3} \mathrm{O}_{12}$ belongs to the family of layered perovskites of bismuth titanates. Its crystal structure can be described by a sandwich of $\left(\mathrm{Bi}_{2} \mathrm{Ti}_{3} \mathrm{O}_{10}\right)^{2-}$ and $\left(\mathrm{Bi}_{2} \mathrm{O}_{2}\right)^{2+}$ layers along the $c$ axis [13-14]. In the literature, it has been widely studied due to its properties as ferroelectric and electro-optic material [26]. On the contrary, $\mathrm{Bi}_{20} \mathrm{TiO}_{32}$ is a much less studied material. The metastable character of this crystalline phase makes it difficult to obtain under mild experimental conditions [18] and it is usually found as impurity 
during the formation of other bismuth titanates; such as $\mathrm{Bi}_{2} \mathrm{Ti}_{2} \mathrm{O}_{7}$ [19]. In the present work, the calcined sample exhibits around $77 \%$ of this metastable phase, even if the preparation method employed a moderate calcination temperature of $400^{\circ} \mathrm{C}$. This will allow obtaining a material with relatively high surface area, as it will be seen later, that is always a desirable feature in catalysis.

Atomic contents of $\mathrm{Bi}, \mathrm{Ti}$ and $\mathrm{O}$ in the samples were stimated by $\mathrm{XRF}$. $\mathrm{Bi} / \mathrm{Ti}$ atomic ratios are shown in Table 1. Fresh sample has a molar ratio $\mathrm{Bi} / \mathrm{Ti}$ of 1.47, not far from the stoichiometric ratio for the phase $\mathrm{Bi}_{4} \mathrm{Ti}_{3} \mathrm{O}_{12}$ which is the only phase seen in its XRD pattern. As it can be observed in Table 1, the same Bi/Ti ratio (1.47) is kept after the calcination of the sample. However, according to XRD pattern for BTO-400 there is a $77 \%$ of $\mathrm{Bi}_{20} \mathrm{TiO}_{32}$ phase, and therefore there is a large excess of $\mathrm{Ti}$ which is not observed in the diffraction pattern; suggesting the presence of amorphous $\mathrm{TiO}_{2}$ which could not be detected by the diffraction technique, according to equation 1 . The presence of amorphous $\mathrm{TiO}_{2}$ will be confirmed by TEM observations as it will be seen later.

$5 \mathrm{Bi}_{4} \mathrm{Ti}_{3} \mathrm{O}_{12} \rightarrow \mathrm{Bi}_{20} \mathrm{TiO}_{32}+14 \mathrm{TiO}_{2} \quad$ (Eq 1.)

Thus, from XRF and XRD results, it can be estimated that BTO-400 is a mixture of three phases, $20 \%$ amorphous $\mathrm{TiO}_{2}, 66 \% \mathrm{Bi}_{20} \mathrm{TiO}_{32}$ and $14 \% \mathrm{Bi}_{4} \mathrm{Ti}_{3} \mathrm{O}_{12}$ (total weight percentages). For the calculation it is assumed that all the $\mathrm{Bi}$ is in one of the two forms of titanate.

Figure 2 depict light absorption spectra for BTO-Fresh and BTO-400 samples; for comparison the spectrum for $\mathrm{TiO}_{2} \mathrm{P} 25$ is also shown. As it can be clearly seen, BTO-400 shows a marked visible light absorption within the visible range up to wavelengths longer than $500 \mathrm{~nm}$, while the spectrum for BTO-Fresh is more similar to the one of 
$\mathrm{TiO}_{2}$ with light absorption mainly in the UV range. From these spectra the band gap energies (BG) of the samples were calculated and the values are shown in Table 1. BTO-Fresh presents a $\mathrm{BG}$ of $2.9 \mathrm{eV}$, slightly lower that the $\mathrm{BG}$ presented by the $\mathrm{TiO}_{2}$ P25 (3.1 eV). On the contrary, BTO-400 presents a much lower value of $2.1 \mathrm{eV}$, which gives account of its visible absorption properties.

Both samples have only one steep absorption edge, which means that the absorption is due to the intrinsic band-gap transition. The narrow band gap of these compounds can be caused for the crystal structure of bismuth titanates, where the valence band consists of a hybridized $\mathrm{Bi} 6 \mathrm{~s}^{2}$ filled orbital and an $\mathrm{O} 2 \mathrm{p}$ orbital [27].

Surface area values (BET) of the samples can also be seen in Table 1. Originally BTOFresh sample presents a BET value of $46 \mathrm{~m}^{2} / \mathrm{g}$, which decreases until $35 \mathrm{~m}^{2} / \mathrm{g}$ after the calcination treatment for BTO-400. Nevertheless, this value is relatively high compared to surface areas shown by other $\mathrm{Bi}_{20} \mathrm{TiO}_{32}$ materials reported in the literature. As mentioned before, $\mathrm{Bi}_{20} \mathrm{TiO}_{32}$ is a metastable phase, difficult to obtain under mild experimental conditions and usually quenching methods are used to approach its synthesis leading to materials with a poor surface area [18]. Even if other materials used as photocatalyst, especially $\mathrm{TiO}_{2}$ based materials, can have much higher surface areas [2], Bi-based materials usually present more moderate ones. Thus, BTO-400 sample with $35 \mathrm{~m}^{2} / \mathrm{g}$ can be considered a material with a relatively high surface area compared to other reported materials of its kind. High surface area is a desired characteristic in the materials used as photocatalysts as increases the number of active sites and promotes the lifetime of photogenerated electron-holes, which could help increasing the efficiency of the photocatalytic process. 
Figure 3 depicts pore diameter for both samples. As it can be observed, for both samples there is a big and wide pore family between 3 and $100 \mathrm{~nm}$. The different pore volume between the calcined and non calcined samples gives account of the different surface area of the materials. Additionally, for BTO-400 sample there is also a smaller pore family to lower pore diameters between 1.5 and $2.5 \mathrm{~nm}$.

Morphology of the samples could be seen by SEM studies. Representative selected pictures of BTO-Fresh and BTO-400 are shown in Figure 4. As it can be observed, samples are formed by particles in the shape of irregular nanosheets, covered by a high number of roundish smaller particles. This coverage seems to be larger in the calcined sample (Fig. 4 B).

The microstructure of BTO-400 sample was analysed by TEM/HRTEM/STEM-HAADFEDX techniques and representative results are presented in Figures 5, 6 and 7. The sample is formed by agglomerated particles (Fig. 5a) with difference features; there are regions formed by large crystals (about $300 \mathrm{~nm}$ ) with a platelet shape (Fig. 5b), and areas formed by very small crystallites $(5-10 \mathrm{~nm})$ with round and elongated shapes (Fig. 5d).

HRTEM was performed to measure the interplanar spacing of the crystals with different shapes. The micrograph presented in Figure $5 \mathrm{c}$, showing one of the large plates, presents interplanar spacing (021) corresponding to the $\mathrm{Bi}_{20} \mathrm{TiO}_{32}$ phase (PDF042-02-02; Tetragonal symmetry; P-421c). This phase was confirmed later by performing EDX analysis.

High resolution images corresponding to the elongated and round crystallites can be seen in Figures $5 e$ and $5 f$ respectively and the interplanar distances indicate that possibly both belong to the $\mathrm{Bi}_{4} \mathrm{Ti}_{3} \mathrm{O}_{12}$ phase (PDF-012-02-13; orthorhombic symmetry; 
Fmmm). The round crystallites are very small with size ranging between 5 and $10 \mathrm{~nm}$ (Fig. 5f) and some interplanar distances could be indexed in the $\mathrm{Bi}_{4} \mathrm{Ti}_{3} \mathrm{O}_{12}$ phase (marked in yellow).

An enlargement of Fig. $5 \mathrm{e}$ is presented in Fig. $5 \mathrm{~g}$ to process the contrast of the elongated crystallites, after obtaining and analyse the First Fourier Transform (FFT) from the three areas marked in pink, it can be deduced that are oriented along the [12, $7,1] ;[9,7,1]$ and $[12,9,1]$ zone axis of the $\mathrm{Bi}_{4} \mathrm{Ti}_{3} \mathrm{O}_{12}$ phase. This fact allows us to conclude without any doubt that these small elongated crystals fit into the $\mathrm{Bi}_{4} \mathrm{Ti}_{3} \mathrm{O}_{12}$ phase. Likewise, some round-faceted crystallites (around $10 \mathrm{~nm}$ ) were found oriented in tangible zone axis as can be observed in Figure 6 . The FFT was performed and the results (Fig. 6d-f) show that the three crystals belong to the $\mathrm{Bi}_{4} \mathrm{Ti}_{3} \mathrm{O}_{12}$ phase and are oriented along the $[9,5,1] ;[9,6,1]$ and $[9,8,1]$ zone axis (the diffracted planes are marked). The crystal borders are very well defined and in the case of Fig. $6 \mathrm{~b}$ a hexagonal shape is distinguished that must be the projection of a polyhedral crystal. EDX analyses were performed with a HAAD detector in SEM mode to check the chemical composition through the sample and a summary of the results are set out in Figure 7. The analysis was carried out with very small beam (about $5 \mathrm{~nm}$ ) giving chemical information of very tiny zones of the sample. The areas where the contrast resembles many different small crystals give a bismuth and titanium atomic percentage (Fig. $7 a-b$ ) that is in agreement with the $\mathrm{Bi}_{4} \mathrm{Ti}_{3} \mathrm{O}_{12}$ phase, which have $\mathrm{Bi} / \mathrm{Ti}$ theoretic atomic percentage of $57 / 43$. The orange marks in the STEM images correspond to the points where the analysis was done. The EDX analysis in the big crystal presented in Fig. 7c (point 1) reveals a $\mathrm{Bi} / \mathrm{Ti}$ atomic percentage of 94/6 indicating that the crystal corresponds to a $\mathrm{Bi}_{20} \mathrm{TiO}_{32}$ phase (theoretic atomic 
percentage of $\mathrm{Bi} / \mathrm{Ti}: 95 / 5)$. The results set of EDX analysis is in good agreement with the XRD and HRTEM results. The atomic percentage values are not very accurate compared to the theoretic for two reasons, first -the two phases are not separated and sometimes the EDX point is located in an area with both chemical compositions and second -the EDX analysis is semiquantitative since standards have not been used.

Finally, it is worth noting that in some areas of BTO-400 sample rounded amorphous particles (Fig. 7d) were found and that the EDX analysis revealed to be titanium dioxide (Fig. 7e). Taking into account that BTO-400 sample was formed from the BTO-Fresh with $\mathrm{Bi}_{4} \mathrm{Ti}_{3} \mathrm{O}_{12}$ composition, part of the transformation of that sample in $\mathrm{Bi}_{20} \mathrm{TiO}_{32}$ composition implies $\mathrm{TiO}_{2}$ formation (Eq 1) and here we have the evidence of titanium dioxide that XRD analysis could not detected it because it is an amorphous phase.

\subsection{Photocatalytic runs}

Before photocatalytic tests, as explained in experimental section, photocatalysts and phenol solutions were allowed to reach adsorption equilibrium in the dark. Phenol adsoption on BTO-Fresh and BTO-400 was similar and not very significant in any case, being just $1.0 \mathrm{ppm}$ and $1.2 \mathrm{ppm}$ of phenol per gram of catalyst, respectively.

Profiles for phenol degradation over BTO-Fresh and BTO-400 are shown in Figure 8, under UV-Vis illumination (Fig. 8 upper figure) and only visible illumination (Fig. 8 lower figure). For comparison purposes the profile over $\mathrm{TiO}_{2}$ Degussa P25 is also shown for both illumination conditions. Figure 9 depicts the corresponding initial velocity rates for all the samples.

With UV-Vis illumination (sunlike emission spectrum), the best photocatalytic performance is found for the $\mathrm{TiO}_{2} \mathrm{P} 25$. However, BTO-400 sample also shows a quite 
good activity for phenol degradation. BTO-Fresh presents a more moderate photocatalytic activity.

A quite different behaviour of the samples is found under visible illumination conditions. Here, BTO-400 shows an outstanding high phenol degradation rate. BTOFresh sample shows a very poor activity, similarly to $\mathrm{TiO}_{2}$ P25 as expected.

It is worth noticing that the BTO-400 material has a visible activity comparable to the UV activity shown by the $\mathrm{TiO}_{2} \mathrm{P} 25$ under similar experimental conditions. This, to the extent of our knowledge, has been rarely reported in the literature.

Calcination of the fresh sample at $400^{\circ} \mathrm{C}$ for $2 \mathrm{~h}$ led to the partial transformation of $\mathrm{Bi}_{4} \mathrm{Ti}_{3} \mathrm{O}_{12}$ into $\mathrm{Bi}_{20} \mathrm{TiO}_{32}$. According to the different characterisation results, a certain amount of amorphous $\mathrm{TiO}_{2}$ is also present. Additionally, TEM study shows a good interconnection of both crystalline phases, which could act as separation paths of the visible photogenerated electro-hole pairs. Good visible light absorption of the BTO-400 is evident from its UV-Vis spectrum and its BG value $(2.1 \mathrm{eV})$.

$\mathrm{Bi}_{4} \mathrm{Ti}_{3} \mathrm{O}_{12}$ has been reported to have good photocatalytic properties under visible illumination [9-12]. However, in our present results the visible activity for this material is rather poor. It seems clear that preparation method has a direct influence in the final properties of the catalyst, and therefore on its photocatalytic activity.

For materials containing $\mathrm{Bi}_{20} \mathrm{TiO}_{32}$ there are not many studies reporting photocatalytic activity. The used preparation methods normally lead to materials with low surface areas, which in catalysis is an important parameter. When phenol degradation rate is estimated for surface area unit of catalyst (Fig. 10), it can be seen that the activity for BTO-400 is even higher that for $\mathrm{TiO}_{2} \mathrm{P} 25$ also under UV-Vis illumination. 
The exceptional visible activity of the BTO-400 material can be then ascribed to different factors. Firstly, the material possesses large light absorption in the visible range, which will allow it to be excited by visible photons. Then, it is composed by two crystalline phases closely interconnected, which could provide an excellent separation path for photogenerated electron-holes pairs thus increasing their lifetimes. This could form a heterostructure coupling between both phases with a more effective separation and transportation of carriers, in a similar manner than systems reported in the literature [28-30]

Then, a relatively high surface area would help by increasing the number of absorption and active sites on the surface of the photocatalyst.

The outstanding visible activity of BTO-400, comparable to the UV activity of $\mathrm{TiO}_{2}$, makes of this material a promising photocatalyst for solar and inner applications of photocatalysis.

\section{Conclusions}

A photocatalyst (namely BTO-400) with high visible activity was obtained by a facile hydrothermal method. The visible activity of this material is of the same magnitude that the UV activity of commercial $\mathrm{TiO}_{2} \mathrm{P} 25$ under the same experimental conditions for phenol degradation. BTO-400 is composed by majority of phase $\mathrm{Bi}_{20} \mathrm{TiO}_{32}(66 \%)$, together with $\mathrm{Bi}_{4} \mathrm{Ti}_{3} \mathrm{O}_{12}$ (14\%) and amorphous $\mathrm{TiO}_{2}$ (20\%). The high visible activity can be ascribed to the characteristic of the material, i.e. high visible light absorption (BG $2.1 \mathrm{eV}$ ), a good separation pathway for visible photogenerated electron-hole pairs due to its characteristic structure and relatively high surface area. All this makes of BTO400 a very promising and suitable material for its use as photocatalyst in solar light and 
inside-room photocatalytic applications. Complementary studies are currently underway.

\section{Acknowledgements}

This work was supported by research fund from Project Ref. CTQ2015-64664-C2-2-P (MINECO/FEDER UE). CITIUS (University of Seville) is acknowledged for XRF measurements.

\section{References}

[1] J. Schneider, M. Matsuoka, M. Takeuchi, J. Zhang, Y. Horiuchi, M. Anpo, D.W. Bahnemann, Understanding $\mathrm{TiO}_{2}$ Photocatalysis: Mechanisms and Materials, Chem. Rev. 114 (2014) 9919-9986.

[2] X. Chen, S.S. Mao, Titanium Dioxide Nanomaterials: Synthesis, Properties, Modifications, and Applications, Chem. Rev. 107 (2007) 2891-2959.

[3] J. Chen, F. Qiu, W. Xu, S. Cao, H. Zhu, Recent progress in enhancing photocatalytic efficiency of $\mathrm{TiO}_{2}$-based materials, Appl. Catal. A 495 (2015) 131140

[4] M.D. Hernandez-Alonso, F. Fresno, S. Suarez, J.M. Coronado, Development of alternative photocatalysts to $\mathrm{TiO}_{2}$ : Challenges and opportunities, Energy Environ. Sci. 2 (2009) 1231-1257

[5] B. Liu, Y. Fang, Z. Li, S. Xu, Visible-Light Nanostructured Photocatalysts--A Review, J. Nanosci. Nanotech. 15 (2015) 889-920. 
[6] H. Peng, J. Lu, C. Wu, Z. Yang, H. Chen, W. Song, P. Li, H. Yin, Co-doped MoS2NPs with matched energy band and low overpotential high efficiently convert $\mathrm{CO}_{2}$ to methanol, Appl. Surf. Sci. 353 (2015) 1003-1012.

[7] T. Wu, L.Liu, M. Pi, D. Zhang, S. Chen, Enhanced magnetic and photocatalytic properties of $\mathrm{Bi}_{2} \mathrm{Fe}_{4} \mathrm{O}_{9}$ semiconductor with large exposed (001) surface, Appl. Surf. Sci. 377 (2016) 253-261.

[8] R. He, J. Zhang, J. Yu, S. Cao, Room-temperature synthesis of BiOl with tailorable ( $\left.\begin{array}{lll}0 & 0 & 1\end{array}\right)$ facets and enhanced photocatalytic activity, J. Colloid Interface Sci. 478 (2016) 201-208.

[9] X. Lin, P. Lv, Q. Guan, H. Li, H. Zhai, C. Liu, Appl. Surf. Sci., Bismuth titanate microspheres: Directed synthesis and their visible light photocatalytic activity, 258 (2012) 7146-7153.

[10] H. He, J. Yin, Y. Li, Y. Zhang, H. Qiu, J. Xu, T. Xu, C. Wang, Size controllable synthesis of single-crystal ferroelectric $\mathrm{Bi}_{4} \mathrm{Ti}_{3} \mathrm{O}_{12}$ nanosheet dominated with $\{00$ 1) facets toward enhanced visible-light-driven photocatalytic activities, Appl. Catal. B 156-157 (2014) 35-43.

[11] P. Hao, Z. Zhao, J. Tian, G. Yu, H. Liu, S. Chen, W. Zhou, Acta Materialia, Bismuth titanate nanobelts through a low-temperature nanoscale solid-state reaction, 62 (2014) 258-266.

[12] W. Zhao, Z. Jia, E. Lei, L. Wang, Z. Li, Y. Dai, J. Phys. Chem. Solids, Photocatalytic degradation efficacy of $\mathrm{Bi}_{4} \mathrm{Ti}_{3} \mathrm{O}_{12}$ micro-scale platelets over methylene blue under visible light, 74 (2013) 1604-1607. 
[13] W.F. Yao, H. Wang, X. H. Xu, S.X. Shang, Y. Hou, Y. Zhang, M. Wang, Synthesis and photocatalytic property of bismuth titanate $\mathrm{Bi}_{4} \mathrm{Ti}_{3} \mathrm{O}_{12}$, Mater. Lett. 57 (2003) 1899-1902.

[14] W.F. Yao, X.H. Xu, H. Wang, J.T. Zhou, X. N. Yang, Y. Zhang, S. X. Shang, B.B. Huang, Photocatalytic property of perovskite bismuth titanate, Appl. Catal. B 52 (2004) 109-116.

[15] L. Xie, J. Ma, H. Tian, J. Zhou, Z. Zhao, P. Wu, Y. Hu, Y. Wang, J. Tao, X. Zhu, Isopropanol-assisted hydrothermal synthesis of bismuth titanate nanophotocatalysts, Mat. Lett. 60 (2006) 284-286.

[16] L. Zheng, G. Yan, Y. Huang, X. Wang, J. Long, L. Li, T. Xu, Visible-light photocatalytic denitrogenation of nitrogen-containing compound in petroleum by metastable $\mathrm{Bi}_{20} \mathrm{TiO}_{32}$, Inter. J. Hydrogen Energy 39 (2014) 13401-13407.

[17] W-N. Su, D.W. Ayele, V. Ochie, C-J. Pan, B-J Hwang, The development of highly crystalline single-phase $\mathrm{Bi}_{20} \mathrm{TiO}_{32}$ nanoparticles for light driven oxygen evolution, Appl. Catal. B 150-151 (2014) 363-369.

[18] H. Cheng, B. Huang, Y. Dai, X. Qin, X. Zhang, Z. Wang, M. Jiang, Visible-light photocatalytic activity of the metastable $\mathrm{Bi}_{20} \mathrm{TiO}_{32}$ synthesized by a hightemperature quenching method, J. Solid State Chem. 182 (2009) 2274-2278.

[19] Y. Hou, M. Wang, X. Xu, H. Wang, S. Shang, D. Wang, W. Yao, $\mathrm{Bi}_{20} \mathrm{TiO}_{32}$ nanocones preprared from Bi-Ti-O mixture by metalorganic decomposition method, J. Crystal Growth 240 (2002) 489-494.

[20] T. Zhou, J. Hu, Mass production and photocatalytic activity of highly crystalline metastable single-phase $\mathrm{Bi}_{20} \mathrm{TiO}_{32}$ nanosheets, Environ. Sci. Technol. 44 (2010) 8698-8703. 
[21] E. Grabowska, J. Reszczyńska, A. Zaleska, Mechanim of phenol photodegradation in the presence of pure and modified- $\mathrm{TiO}_{2}$ : A review, Water Res. 46 (2012) 54535471.

[22] G. Busca, S. Berardinelli, C. Resini, L. Arrighi, Technologies for the removal of phenol from fluid streams: A short review of recent developments, J. Hazardous. Mater. 160 (2008) 265-288.

[23] M. Rochkind, S. Pasternak, Y. Paz, Using Dyes for Evaluating Photocatalytic Properties: A Critical Review, Molecules 20 (2015) 88-110.

[24] S. Murcia-López, M.C. Hidalgo, J.A. Navío, Synthesis, characterization and photocatalytic activity of $\mathrm{Bi}$-doped $\mathrm{TiO}_{2}$ photocatalysts under simulated solar irradiation, Appl. Catal. A 404 (2011) 59-67.

[25] M.C. Hidalgo, G. Colón, J.A. Navío, Modification of the physicochemical properties of commercial $\mathrm{TiO}_{2}$ samples by soft mechanical activation, J. Photochem. Photobiol. A 148 (2002) 341-348.

[26] A.Q. Jiang, H.G. Li, L.D. Zhang, Dielectric study in nanocrystalline $\mathrm{Bi}_{4} \mathrm{Ti}_{3} \mathrm{O}_{12}$ prepared by chemical coprecipitation, J. Appl. Phys. 83 (1998) 4878-4883.

[27] R. He, S. Cao, P. Zhou, J. Yu, Recent advances in visible light Bi-based photocatalysts, Chin. J. Catal. 35 (2014) 989-1007.

[28] X. Zhang, L. Zhang, J-S. Hu, C-L- Pan, C-M. Hou, Facile hydrothermal synthesis of novel $\mathrm{Bi}_{12} \mathrm{TiO}_{20}-\mathrm{Bi}_{2} \mathrm{WO}_{6}$ heterostructure photocatalyst with enhanced photocatalytic activity, Appl. Surf. Sci. 346 (2015) 33-40.

[29] F. Duo, Y. Wang, X. Mao, X. Zhang, Y. Wang, C. Fan, A BiPO ${ }_{4} / \mathrm{BiOCl}$ heterojunction photocatalyst with enhancedelectron-hole separation and excellent photocatalytic performance, Appl. Surf. Sci. 340 (2015) 35-42. 
[30] Y. Deng, L. tang, G. Zeng, H. Dong, M. Yan, J. Wang, W. Hu, J. Wang, Y. Zhou, J. Tang, Enhanced visible light photocatalytic performance of polyaniline modified mesoporous single Crystal TiO2 microsphere, Appl. Surf. Sci. 387 (2016) 882-893. 


\section{Figures Captions}

Figure 1. XRD patterns for $\mathrm{TiO}_{2} \mathrm{P} 25, \mathrm{BTO}-$ Fresh and $\mathrm{BTO}-400$ samples. "*” indicates $\mathrm{Bi}_{4} \mathrm{Ti}_{3} \mathrm{O}_{12}$ diffraction peaks (PDF-012-02-13) and “ $\Theta$ " indicates $\mathrm{Bi}_{20} \mathrm{TiO}_{32}$ peaks (PDF042-02-02).

Figure 2. UV-Vis DR spectra for BTO-400 (blue line), BTO-Fresh (black line) and $\mathrm{TiO}_{2}$ P25 (red line).

Figure 3. Pore size distributions for BTO-Fresh (red line) and BTO-400 (black line) samples.

Figure 4. SEM pictures of BTO-Fresh (A) and BTO-400 (B).

Figure 5. TEM images of BTO-400 sample presenting different areas (a, b and $\mathbf{d})$; HRTEM micrographs showing the interplanar spacing of the two phases, $\mathrm{Bi}_{20} \mathrm{TiO}_{32}$ and $\mathrm{Bi}_{4} \mathrm{Ti}_{3} \mathrm{O}_{12}$ (c, e and $\mathbf{f}$ ) and enlargement of the image presented in $\boldsymbol{e}$, the insets correspond to the FFTs of the square marked areas $(\boldsymbol{g})$.

Figure 6. HRTEM micrographs of the BTO-400 sample and the corresponding FFTs of the square marked areas showing three different zone axes of the $\mathrm{Bi}_{4} \mathrm{Ti}_{3} \mathrm{O}_{12}$ phase.

Figure 7. STEM images of BTO-400 sample obtained from different areas and showing the two positions where the EDX analysis was done ( $\mathbf{a}, \mathbf{b}$ and $\mathbf{c})$. TEM image (d) and STEM image of the same region where two positions were analysed by EDX (e) and EDX spectra obtained from the two positions( $\mathbf{f}$ and $\mathbf{g})$.

Figure 8. Phenol photodegradation profiles over the different photocatalysts under UV-Vis illumination (upper figure) and visible illumination (lower figure).

Figure 9. Initial reaction rates for phenol photodegradation over the different photocatalysts under UV-Visible (left) and just Visible illumination (right). 
Figure 10. Initial reaction rates per surface area unit of photocatalyst for phenol photodegradation under UV-Visible (left) and just Visible illumination (right). 


\title{
Outstanding visible photocatalytic activity of a new mixed
}

\section{Bismuth Titanatate material}

\author{
P. Zambrano ${ }^{1,2}$, M.J. Sayagués ${ }^{1}$, J.A. Navío ${ }^{1}$ and M.C. Hidalgo ${ }^{1 *}$ \\ ${ }^{1}$ Instituto de Ciencia de Materiales de Sevilla, CSIC-Universidad de Sevilla, Americo \\ Vespucio 49, 410092, Sevilla, Spain \\ ${ }^{2}$ Departamento Cristalografía, Mineralogía y Química Agrícola, Universidad de Sevilla, \\ C/Profesor García González s/n, 41012 Sevilla, Spain
}

\begin{abstract}
In this work, a new photocatalyst based on bismuth titanates with outstanding visible photocatalytic activity was prepared by a facile hydrothermal method. The synthesised material showed visible activity as high as UV activity of commercial $\mathrm{TiO}_{2}$ P25 under the same experimental conditions for phenol degradation. A wide characterisation of the photocatalyst was performed. The material was composed of three phases; majority of $\mathrm{Bi}_{20} \mathrm{TiO}_{32}$ closely interconnected to $\mathrm{Bi}_{4} \mathrm{Ti}_{3} \mathrm{O}_{12}$ and amorphous $\mathrm{TiO}_{2}$. The high visible activity showed by this material could be ascribed to a combination of several features; i.e. low band gap energy value $(2.1 \mathrm{eV})$, a structure allowing a good separation path for visible photogenerated electron-holes pairs and a relatively high surface area. This photocatalyst appeared as a promising material for solar and visible applications of photocatalysis.
\end{abstract}

Keywords: visible photocatalysis, $\mathrm{Bi}_{4} \mathrm{Ti}_{3} \mathrm{O}_{12}, \mathrm{Bi}_{20} \mathrm{TiO}_{32}$, phenol degradation

\footnotetext{
Corresponding author: Tel: +34954 489501

*E-mail: carmen.hidalgo@csic.es
} 


\section{Introduction}

Titanium dioxide $\left(\mathrm{TiO}_{2}\right)$ has been until now the most studied material used as photocatalyst, due to its good properties such as nontoxicity, chemical and photochemical stability and remarkable photoactivity under ultraviolet irradiation [12]. However, there are also some drawbacks of this material which hinder its wide use for many applications; mainly its high recombination rate of photogenerated electronholes which lower its photoefficiency and its inability to be active under visible light. Due to its large band-gap $\mathrm{TiO}_{2}$ can only absorb light in the ultraviolet region which limits its implementation for solar and indoor applications [3]. Thus, in the last years, a lot of effort in the photocatalysis research has been focused in the development and preparation of alternative materials to $\mathrm{TiO}_{2}$ for solar and visible photocatalytic applications [4-6]. In this sense the searching of new or modified materials with absorption in the visible range of the spectrum has been a deal in many studies with the objective of obtaining materials with also a lower recombination rate of photogenerated charges for getting higher efficiency in the photocatalytic processes [4-8].

In this context, the family of bismuth titanates has attracted the interest of researchers in the area due to promising positive results in visible photocatalysis. Bismuth titanates include several different phases such as $\mathrm{Bi}_{2} \mathrm{Ti}_{2} \mathrm{O}_{7}, \mathrm{Bi}_{2} \mathrm{Ti}_{4} \mathrm{O}_{11}, \mathrm{Bi}_{4} \mathrm{Ti}_{3} \mathrm{O}_{12}, \mathrm{Bi}_{12} \mathrm{TiO}_{20}$, $\mathrm{Bi}_{20} \mathrm{TiO}_{32}$, etc. [9]. Probably the most studied phase, for photocatalytic applications, is the $\mathrm{Bi}_{4} \mathrm{Ti}_{3} \mathrm{O}_{12}$, which has shown high or moderate photocatalytic activity under visible illumination in several studies [10-15]. Although the phase $\mathrm{Bi}_{20} \mathrm{TiO}_{32}$ has also appeared as photoactive, it has been much less studied due to its metastable character, which makes it difficult to obtain as single phase or by means of mild conditions preparation 
methods. The visible activity of $\mathrm{Bi}_{20} \mathrm{TiO}_{32}$ is ascribed to the hybridized valence band by Bi6s and 02p orbitals which lead to a low band gap value [16]. This phase has been mainly obtained by synthetic procedures involving severe conditions as quenching methods at high temperatures [17-20]. This kind of preparation methods usually gives low surface areas and highly irregular materials, which in principle are negative features for catalysts. Therefore, when these materials are expected to be used as photocatalysts it would be worth to study alternative synthetic methods to get this kind of materials using mild preparation conditions [20].

In this paper, materials based in $\mathrm{Bi}-\mathrm{Ti}-\mathrm{O}$ have been prepared with an extraordinary activity in the visible, evaluated in the phenol photodegradation reaction. This model compound was chosen due to its wide utilisation in environmental photocatalytic studies [21-22]. Additionally, as visible activity was also evaluated, it appears as more appropriate substrate than dies, which have been also often used but that could present photosensibilisation effects masking real visible activity [23].

A wide characterization has been performed in order to understand the good visible photocatalytic performance. As reference material $\mathrm{TiO}_{2}$ Degussa P25 was used and it was observed that the visible activity of the obtained Bi-Ti-O material was comparable to the activity of $\mathrm{TiO}_{2} \mathrm{P} 25$ under the ultraviolet illumination under the same experimental parameters. This, as far as we know, has not often been reported. The materials obtained are then very promising for any kind of solar application and/or indoors application, where visible light would be the source of illumination.

\section{Experimental}

\subsection{Catalysts preparation}


The Bi-Ti-O sample was prepared by mixing two solutions with the corresponding precursors and a further hydrothermal treatment.

First solution (solution A) was obtained by mixing Titanium tetraisopropoxide (TTiP Aldrich 97\%) in Isopropanol (Aldrich 99.9\%) and distilled water. This solution was kept under stirring at room temperature for one week to obtain a milky sol, as described in a previous paper [24].

Second solution (solution $\mathrm{B}$ ) was prepared by dissolving $\mathrm{Bi}\left(\mathrm{NO}_{3}\right)_{3} \cdot 5 \mathrm{H}_{2} \mathrm{O}$ (analytical grade from Aldrich) in glacial acetic acid (reactive grade from Aldrich) to obtain a $1 \mathrm{M}$ solution.

Then, solution B was slowly added to solution A and kept under stirring for one hour. Afterwards, triethylamine (TEA, Aldrich $>99.5 \%$ ) was added until reaching a $\mathrm{pH}$ value of 9. The resulting sol was transferred into a Teflon recipient inside of a stainless steel autoclave and hydrothermal treatment was performed at $140 \circ \mathrm{C}$ for 20 hours. A precipitate was obtained and was then filtered, repeatedly washed with distilled water and dried overnight at $120^{\circ} \mathrm{C}$.

The obtained powder was divided in two portions; one of them was calcined at $400^{\circ} \mathrm{C}$ for 2 hours. Hereafter, the non-calcined sample is named as BTO-Fresh, and the calcined one BTO-400.

On the other hand, commercial $\mathrm{TiO}_{2}$ Degussa P25 was employed "as received" without previous treatment.

\subsection{Characterisation of the catalysts}


Crystalline phase composition and degree of crystallinity of the samples were estimated by X-ray diffraction (XRD), on a Siemens D-501 diffractometer with Ni filter and graphite monochromator using $\mathrm{Cu}$ Ka radiation (0.15406 nm).

Global composition was determined by X-ray fluorescence spectrometry (XRF) in a Panalytical Axios sequential spectrophotometer with a rhodium tube as the source of radiation. XRF measurements were performed onto pressed pellets (sample included in $10 w t \%$ of wax).

BET surface area and porosity measurements were carried out by $\mathrm{N}_{2}$ adsorption at $77 \mathrm{~K}$ using a Micromeritics 2010 instrument.

The morphology and microcharacterization of the samples were analyzed using scanning (SEM) and transmission (TEM) electron microscopy techniques. The samples were dispersed in ethanol using an ultrasonicator and dropped on a carbon coated copper grid. For SEM observations, a Hitachi S 4800 field emission-SEM was used in secondary electron mode at an acceleration voltage of $5 \mathrm{kV}$ with an EDX detector for chemical analysis. The samples were sufficiently conductive, thus they were not coated with any conductive material in order to avoid charging artefacts.

For TEM studies, a FEI TECNAI G2 F30 S-twin high resolution transmission electron microscope (HRTEM) was used, with field emission gun (FEG) and scanningtransmission capabilities (STEM) operating at $300 \mathrm{kV}$ and $0.2 \mathrm{~nm}$ point resolution. The microscope was equipped with a high angle annular dark field (HAADF) detector with $0.16 \mathrm{~nm}$ point resolution from Fischione Instruments, and an INCA X-Max 80 silicon drift detector (SDD) for the energy dispersive X-ray analysis (EDX). The Gatan Digital Micrograph software (Gatan Inc.) was used to measure lattice spacing and to calculate the First Fourier Transform (FFT) and the Java version Electron Microscope Software 
(JEM) was used to analysed the different phases of the samples. The analysis of the HAAD-STEM images and the EDX spectra profile were done with the ES Vision software (FEI Company).

UV-vis spectra were recorded by using a Cary 100 (Varian) in the diffuse reflectance mode $(R)$ and transformed to a magnitude proportional to the extinction coefficient $(K)$ through the Kubelka-Munk function, $F(R \infty)$. Samples were mixed with $\mathrm{BaSO}_{4}$ that does not absorb in the UV-vis radiation range (white standard). Scans range was $240-800$ $\mathrm{nm}$.

\subsection{Photocatalytic activity}

The photocatalytic activity of the samples was evaluated in the phenol oxidation reaction using a batch reactor $(250 \mathrm{ml})$ and an Osram Ultra-Vitalux lamp $(300 \mathrm{~W})$ with sunlike radiation spectrum. A concentration of $1 \mathrm{~g} / \mathrm{L}$ of photocatalyst was used and the initial concentration of phenol was $50 \mathrm{ppm}$.

The intensity of the incident UVA light on the solution was measured with a PMA 2200 UVA photometer (Solar Light Co.) being ca. $90 \mathrm{~W} / \mathrm{m}^{2}$ (UVA PMA2110 sensor; spectral response $320-400 \mathrm{~nm}$ ). On the other hand, the intensity of light in the visible range measured in this case was $110 \mathrm{~W} / \mathrm{m}^{2}$ (Photopic PMA21300 sensor; spectral response 400-700 nm). The photocatalytic runs in the visible range were performed by using a polyester UV filter sheet (Edmund Optics) showing 99.9\% of absorbance below 390 $\mathrm{nm}$.

In the oxidation tests, an oxygen flow was employed what produces a homogenous suspension of the catalyst in the solution. Before each experiment, the catalysts were 
settled in suspension with the reagent mixture for $20 \mathrm{~min}$ to allow catalyst-phenol adsorption equilibration.

The evolution of the phenol concentration was followed through HPLC analysis (Agilent, 1200 Serie) using filtered aliquots ca. $2 \mathrm{ml}$ of the suspension (Millipore Millex25 $0.45 \mathrm{~mm}$ membrane filter).

Blank experiments were performed in the dark with catalyst as well as with illumination and no catalyst, and no changes in the initial concentration of phenol were observed in any of both cases.

\section{Results and discussion}

\subsection{Characterisation}

Figure 1 show XRD patterns of the fresh sample and after calcination treatment. As it can be seen, the sample is already crystalline before calcination and the only phase present can be identified as $\mathrm{Bi}_{4} \mathrm{Ti}_{3} \mathrm{O}_{12}$ (PDF-012-02-13). After calcination at 400 ㅇ $\mathrm{C}$ for 2h, two crystalline phases can be identified, i.e $\mathrm{Bi}_{20} \mathrm{TiO}_{32}$ (PDF-042-02-02) and $\mathrm{Bi}_{4} \mathrm{Ti}_{3} \mathrm{O}_{12}$; with estimated percentages of $77 \%$ and $23 \%$ respectively (see Table 1 ). For comparison XRD pattern of commercial $\mathrm{TiO}_{2}$ Degussa P25 is also shown, where anatase and rutile phases of titania can be seen in a ratio 80:20 respectively [25].

$\mathrm{Bi}_{4} \mathrm{Ti}_{3} \mathrm{O}_{12}$ belongs to the family of layered perovskites of bismuth titanates. Its crystal structure can be described by a sandwich of $\left(\mathrm{Bi}_{2} \mathrm{Ti}_{3} \mathrm{O}_{10}\right)^{2-}$ and $\left(\mathrm{Bi}_{2} \mathrm{O}_{2}\right)^{2+}$ layers along the $c$ axis [13-14]. In the literature, it has been widely studied due to its properties as ferroelectric and electro-optic material [26]. On the contrary, $\mathrm{Bi}_{20} \mathrm{TiO}_{32}$ is a much less studied material. The metastable character of this crystalline phase makes it difficult to obtain under mild experimental conditions [18] and it is usually found as impurity 
during the formation of other bismuth titanates; such as $\mathrm{Bi}_{2} \mathrm{Ti}_{2} \mathrm{O}_{7}$ [19]. In the present work, the calcined sample exhibits around $77 \%$ of this metastable phase, even if the preparation method employed a moderate calcination temperature of $400^{\circ} \mathrm{C}$. This will allow obtaining a material with relatively high surface area, as it will be seen later, that is always a desirable feature in catalysis.

Atomic contents of $\mathrm{Bi}, \mathrm{Ti}$ and $\mathrm{O}$ in the samples were stimated by $\mathrm{XRF}$. $\mathrm{Bi} / \mathrm{Ti}$ atomic ratios are shown in Table 1. Fresh sample has a molar ratio $\mathrm{Bi} / \mathrm{Ti}$ of 1.47 , not far from the stoichiometric ratio for the phase $\mathrm{Bi}_{4} \mathrm{Ti}_{3} \mathrm{O}_{12}$ which is the only phase seen in its XRD pattern. As it can be observed in Table 1, the same Bi/Ti ratio (1.47) is kept after the calcination of the sample. However, according to XRD pattern for BTO-400 there is a $77 \%$ of $\mathrm{Bi}_{20} \mathrm{TiO}_{32}$ phase, and therefore there is a large excess of $\mathrm{Ti}$ which is not observed in the diffraction pattern; suggesting the presence of amorphous $\mathrm{TiO}_{2}$ which could not be detected by the diffraction technique, according to equation 1 . The presence of amorphous $\mathrm{TiO}_{2}$ will be confirmed by TEM observations as it will be seen later.

$5 \mathrm{Bi}_{4} \mathrm{Ti}_{3} \mathrm{O}_{12} \rightarrow \mathrm{Bi}_{20} \mathrm{TiO}_{32}+14 \mathrm{TiO}_{2} \quad$ (Eq 1.)

Thus, from XRF and XRD results, it can be estimated that BTO-400 is a mixture of three phases, $20 \%$ amorphous $\mathrm{TiO}_{2}, 66 \% \mathrm{Bi}_{20} \mathrm{TiO}_{32}$ and $14 \% \mathrm{Bi}_{4} \mathrm{Ti}_{3} \mathrm{O}_{12}$ (total weight percentages). For the calculation it is assumed that all the $\mathrm{Bi}$ is in one of the two forms of titanate.

Figure 2 depict light absorption spectra for BTO-Fresh and BTO-400 samples; for comparison the spectrum for $\mathrm{TiO}_{2} \mathrm{P} 25$ is also shown. As it can be clearly seen, BTO-400 shows a marked visible light absorption within the visible range up to wavelengths longer than $500 \mathrm{~nm}$, while the spectrum for BTO-Fresh is more similar to the one of 
$\mathrm{TiO}_{2}$ with light absorption mainly in the UV range. From these spectra the band gap energies (BG) of the samples were calculated and the values are shown in Table 1. BTO-Fresh presents a $\mathrm{BG}$ of $2.9 \mathrm{eV}$, slightly lower that the $\mathrm{BG}$ presented by the $\mathrm{TiO}_{2}$ P25 (3.1 eV). On the contrary, BTO-400 presents a much lower value of $2.1 \mathrm{eV}$, which gives account of its visible absorption properties.

Both samples have only one steep absorption edge, which means that the absorption is due to the intrinsic band-gap transition. The narrow band gap of these compounds can be caused for the crystal structure of bismuth titanates, where the valence band consists of a hybridized $\mathrm{Bi} 6 \mathrm{~s}^{2}$ filled orbital and an $\mathrm{O} 2 \mathrm{p}$ orbital [27].

Surface area values (BET) of the samples can also be seen in Table 1. Originally BTOFresh sample presents a BET value of $46 \mathrm{~m}^{2} / \mathrm{g}$, which decreases until $35 \mathrm{~m}^{2} / \mathrm{g}$ after the calcination treatment for BTO-400. Nevertheless, this value is relatively high compared to surface areas shown by other $\mathrm{Bi}_{20} \mathrm{TiO}_{32}$ materials reported in the literature. As mentioned before, $\mathrm{Bi}_{20} \mathrm{TiO}_{32}$ is a metastable phase, difficult to obtain under mild experimental conditions and usually quenching methods are used to approach its synthesis leading to materials with a poor surface area [18]. Even if other materials used as photocatalyst, especially $\mathrm{TiO}_{2}$ based materials, can have much higher surface areas [2], Bi-based materials usually present more moderate ones. Thus, BTO-400 sample with $35 \mathrm{~m}^{2} / \mathrm{g}$ can be considered a material with a relatively high surface area compared to other reported materials of its kind. High surface area is a desired characteristic in the materials used as photocatalysts as increases the number of active sites and promotes the lifetime of photogenerated electron-holes, which could help increasing the efficiency of the photocatalytic process. 
Figure 3 depicts pore diameter for both samples. As it can be observed, for both samples there is a big and wide pore family between 3 and $100 \mathrm{~nm}$. The different pore volume between the calcined and non calcined samples gives account of the different surface area of the materials. Additionally, for BTO-400 sample there is also a smaller pore family to lower pore diameters between 1.5 and $2.5 \mathrm{~nm}$.

Morphology of the samples could be seen by SEM studies. Representative selected pictures of BTO-Fresh and BTO-400 are shown in Figure 4. As it can be observed, samples are formed by particles in the shape of irregular nanosheets, covered by a high number of roundish smaller particles. This coverage seems to be larger in the calcined sample (Fig. 4 B).

The microstructure of BTO-400 sample was analysed by TEM/HRTEM/STEM-HAADFEDX techniques and representative results are presented in Figures 5, 6 and 7. The sample is formed by agglomerated particles (Fig. 5a) with difference features; there are regions formed by large crystals (about $300 \mathrm{~nm}$ ) with a platelet shape (Fig. 5b), and areas formed by very small crystallites $(5-10 \mathrm{~nm})$ with round and elongated shapes (Fig. 5d).

HRTEM was performed to measure the interplanar spacing of the crystals with different shapes. The micrograph presented in Figure $5 c$, showing one of the large plates, presents interplanar spacing (021) corresponding to the $\mathrm{Bi}_{20} \mathrm{TiO}_{32}$ phase (PDF042-02-02; Tetragonal symmetry; P-421c). This phase was confirmed later by performing EDX analysis.

High resolution images corresponding to the elongated and round crystallites can be seen in Figures $5 e$ and $5 f$ respectively and the interplanar distances indicate that possibly both belong to the $\mathrm{Bi}_{4} \mathrm{Ti}_{3} \mathrm{O}_{12}$ phase (PDF-012-02-13; orthorhombic symmetry; 
Fmmm). The round crystallites are very small with size ranging between 5 and $10 \mathrm{~nm}$ (Fig. 5f) and some interplanar distances could be indexed in the $\mathrm{Bi}_{4} \mathrm{Ti}_{3} \mathrm{O}_{12}$ phase (marked in yellow).

An enlargement of Fig. $5 \mathrm{e}$ is presented in Fig. $5 \mathrm{~g}$ to process the contrast of the elongated crystallites, after obtaining and analyse the First Fourier Transform (FFT) from the three areas marked in pink, it can be deduced that are oriented along the [12, $7,1] ;[9,7,1]$ and $[12,9,1]$ zone axis of the $\mathrm{Bi}_{4} \mathrm{Ti}_{3} \mathrm{O}_{12}$ phase. This fact allows us to conclude without any doubt that these small elongated crystals fit into the $\mathrm{Bi}_{4} \mathrm{Ti}_{3} \mathrm{O}_{12}$ phase. Likewise, some round-faceted crystallites (around $10 \mathrm{~nm}$ ) were found oriented in tangible zone axis as can be observed in Figure 6 . The FFT was performed and the results (Fig. 6d-f) show that the three crystals belong to the $\mathrm{Bi}_{4} \mathrm{Ti}_{3} \mathrm{O}_{12}$ phase and are oriented along the $[9,5,1] ;[9,6,1]$ and $[9,8,1]$ zone axis (the diffracted planes are marked). The crystal borders are very well defined and in the case of Fig. $6 \mathrm{~b}$ a hexagonal shape is distinguished that must be the projection of a polyhedral crystal. EDX analyses were performed with a HAAD detector in SEM mode to check the chemical composition through the sample and a summary of the results are set out in Figure 7. The analysis was carried out with very small beam (about $5 \mathrm{~nm}$ ) giving chemical information of very tiny zones of the sample. The areas where the contrast resembles many different small crystals give a bismuth and titanium atomic percentage (Fig. 7a-b) that is in agreement with the $\mathrm{Bi}_{4} \mathrm{Ti}_{3} \mathrm{O}_{12}$ phase, which have $\mathrm{Bi} / \mathrm{Ti}$ theoretic atomic percentage of $57 / 43$. The orange marks in the STEM images correspond to the points where the analysis was done. The EDX analysis in the big crystal presented in Fig. 7c (point 1) reveals a Bi/Ti atomic percentage of 94/6 indicating that the crystal corresponds to a $\mathrm{Bi}_{20} \mathrm{TiO}_{32}$ phase (theoretic atomic 
percentage of $\mathrm{Bi} / \mathrm{Ti}: 95 / 5)$. The results set of EDX analysis is in good agreement with the XRD and HRTEM results. The atomic percentage values are not very accurate compared to the theoretic for two reasons, first -the two phases are not separated and sometimes the EDX point is located in an area with both chemical compositions and second -the EDX analysis is semiquantitative since standards have not been used.

Finally, it is worth noting that in some areas of BTO-400 sample rounded amorphous particles (Fig. 7d) were found and that the EDX analysis revealed to be titanium dioxide (Fig. 7e). Taking into account that BTO-400 sample was formed from the BTO-Fresh with $\mathrm{Bi}_{4} \mathrm{Ti}_{3} \mathrm{O}_{12}$ composition, part of the transformation of that sample in $\mathrm{Bi}_{20} \mathrm{TiO}_{32}$ composition implies $\mathrm{TiO}_{2}$ formation (Eq 1) and here we have the evidence of titanium dioxide that XRD analysis could not detected it because it is an amorphous phase.

\subsection{Photocatalytic runs}

Before photocatalytic tests, as explained in experimental section, photocatalysts and phenol solutions were allowed to reach adsorption equilibrium in the dark. Phenol adsoption on BTO-Fresh and BTO-400 was similar and not very significant in any case, being just $1.0 \mathrm{ppm}$ and $1.2 \mathrm{ppm}$ of phenol per gram of catalyst, respectively.

Profiles for phenol degradation over BTO-Fresh and BTO-400 are shown in Figure 8, under UV-Vis illumination (Fig. 8 upper figure) and only visible illumination (Fig. 8 lower figure). For comparison purposes the profile over $\mathrm{TiO}_{2}$ Degussa P25 is also shown for both illumination conditions. Figure 9 depicts the corresponding initial velocity rates for all the samples.

With UV-Vis illumination (sunlike emission spectrum), the best photocatalytic performance is found for the $\mathrm{TiO}_{2} \mathrm{P} 25$. However, BTO-400 sample also shows a quite 
good activity for phenol degradation. BTO-Fresh presents a more moderate photocatalytic activity.

A quite different behaviour of the samples is found under visible illumination conditions. Here, BTO-400 shows an outstanding high phenol degradation rate. BTOFresh sample shows a very poor activity, similarly to $\mathrm{TiO}_{2}$ P25 as expected.

It is worth noticing that the BTO-400 material has a visible activity comparable to the UV activity shown by the $\mathrm{TiO}_{2} \mathrm{P} 25$ under similar experimental conditions. This, to the extent of our knowledge, has been rarely reported in the literature.

Calcination of the fresh sample at $400^{\circ} \mathrm{C}$ for $2 \mathrm{~h}$ led to the partial transformation of $\mathrm{Bi}_{4} \mathrm{Ti}_{3} \mathrm{O}_{12}$ into $\mathrm{Bi}_{20} \mathrm{TiO}_{32}$. According to the different characterisation results, a certain amount of amorphous $\mathrm{TiO}_{2}$ is also present. Additionally, TEM study shows a good interconnection of both crystalline phases, which could act as separation paths of the visible photogenerated electro-hole pairs. Good visible light absorption of the BTO-400 is evident from its UV-Vis spectrum and its BG value $(2.1 \mathrm{eV})$.

$\mathrm{Bi}_{4} \mathrm{Ti}_{3} \mathrm{O}_{12}$ has been reported to have good photocatalytic properties under visible illumination [9-12]. However, in our present results the visible activity for this material is rather poor. It seems clear that preparation method has a direct influence in the final properties of the catalyst, and therefore on its photocatalytic activity.

For materials containing $\mathrm{Bi}_{20} \mathrm{TiO}_{32}$ there are not many studies reporting photocatalytic activity. The used preparation methods normally lead to materials with low surface areas, which in catalysis is an important parameter. When phenol degradation rate is estimated for surface area unit of catalyst (Fig. 10), it can be seen that the activity for BTO-400 is even higher that for $\mathrm{TiO}_{2}$ P25 also under UV-Vis illumination. 
The exceptional visible activity of the BTO-400 material can be then ascribed to different factors. Firstly, the material possesses large light absorption in the visible range, which will allow it to be excited by visible photons. Then, it is composed by two crystalline phases closely interconnected, which could provide an excellent separation path for photogenerated electron-holes pairs thus increasing their lifetimes. This could form a heterostructure coupling between both phases with a more effective separation and transportation of carriers, in a similar manner than systems reported in the literature [28-30]

Then, a relatively high surface area would help by increasing the number of absorption and active sites on the surface of the photocatalyst.

The outstanding visible activity of BTO-400, comparable to the UV activity of $\mathrm{TiO}_{2}$, makes of this material a promising photocatalyst for solar and inner applications of photocatalysis.

\section{Conclusions}

A photocatalyst (namely BTO-400) with high visible activity was obtained by a facile hydrothermal method. The visible activity of this material is of the same magnitude that the UV activity of commercial $\mathrm{TiO}_{2} \mathrm{P} 25$ under the same experimental conditions for phenol degradation. BTO-400 is composed by majority of phase $\mathrm{Bi}_{20} \mathrm{TiO}_{32}(66 \%)$, together with $\mathrm{Bi}_{4} \mathrm{Ti}_{3} \mathrm{O}_{12}(14 \%)$ and amorphous $\mathrm{TiO}_{2}$ (20\%). The high visible activity can be ascribed to the characteristic of the material, i.e. high visible light absorption (BG $2.1 \mathrm{eV}$ ), a good separation pathway for visible photogenerated electron-hole pairs due to its characteristic structure and relatively high surface area. All this makes of BTO400 a very promising and suitable material for its use as photocatalyst in solar light and 
inside-room photocatalytic applications. Complementary studies are currently underway.

\section{Acknowledgements}

This work was supported by research fund from Project Ref. CTQ2015-64664-C2-2-P (MINECO/FEDER UE). CITIUS (University of Seville) is acknowledged for XRF measurements.

\section{References}

[1] J. Schneider, M. Matsuoka, M. Takeuchi, J. Zhang, Y. Horiuchi, M. Anpo, D.W. Bahnemann, Understanding $\mathrm{TiO}_{2}$ Photocatalysis: Mechanisms and Materials, Chem. Rev. 114 (2014) 9919-9986.

[2] X. Chen, S.S. Mao, Titanium Dioxide Nanomaterials: Synthesis, Properties, Modifications, and Applications, Chem. Rev. 107 (2007) 2891-2959.

[3] J. Chen, F. Qiu, W. Xu, S. Cao, H. Zhu, Recent progress in enhancing photocatalytic efficiency of $\mathrm{TiO}_{2}$-based materials, Appl. Catal. A 495 (2015) 131140

[4] M.D. Hernandez-Alonso, F. Fresno, S. Suarez, J.M. Coronado, Development of alternative photocatalysts to $\mathrm{TiO}_{2}$ : Challenges and opportunities, Energy Environ. Sci. 2 (2009) 1231-1257

[5] B. Liu, Y. Fang, Z. Li, S. Xu, Visible-Light Nanostructured Photocatalysts--A Review, J. Nanosci. Nanotech. 15 (2015) 889-920. 
[6] H. Peng, J. Lu, C. Wu, Z. Yang, H. Chen, W. Song, P. Li, H. Yin, Co-doped MoS2NPs with matched energy band and low overpotential high efficiently convert $\mathrm{CO}_{2}$ to methanol, Appl. Surf. Sci. 353 (2015) 1003-1012.

[7] T. Wu, L.Liu, M. Pi, D. Zhang, S. Chen, Enhanced magnetic and photocatalytic properties of $\mathrm{Bi}_{2} \mathrm{Fe}_{4} \mathrm{O}_{9}$ semiconductor with large exposed (001) surface, Appl. Surf. Sci. 377 (2016) 253-261.

[8] R. He, J. Zhang, J. Yu, S. Cao, Room-temperature synthesis of BiOl with tailorable $\left(\begin{array}{lll}0 & 0 & 1\end{array}\right)$ facets and enhanced photocatalytic activity, J. Colloid Interface Sci. 478 (2016) 201-208.

[9] X. Lin, P. Lv, Q. Guan, H. Li, H. Zhai, C. Liu, Appl. Surf. Sci., Bismuth titanate microspheres: Directed synthesis and their visible light photocatalytic activity, 258 (2012) 7146-7153.

[10] H. He, J. Yin, Y. Li, Y. Zhang, H. Qiu, J. Xu, T. Xu, C. Wang, Size controllable synthesis of single-crystal ferroelectric $\mathrm{Bi}_{4} \mathrm{Ti}_{3} \mathrm{O}_{12}$ nanosheet dominated with $\{00$ 1\} facets toward enhanced visible-light-driven photocatalytic activities, Appl. Catal. B 156-157 (2014) 35-43.

[11] P. Hao, Z. Zhao, J. Tian, G. Yu, H. Liu, S. Chen, W. Zhou, Acta Materialia, Bismuth titanate nanobelts through a low-temperature nanoscale solid-state reaction, 62 (2014) 258-266.

[12] W. Zhao, Z. Jia, E. Lei, L. Wang, Z. Li, Y. Dai, J. Phys. Chem. Solids, Photocatalytic degradation efficacy of $\mathrm{Bi}_{4} \mathrm{Ti}_{3} \mathrm{O}_{12}$ micro-scale platelets over methylene blue under visible light, 74 (2013) 1604-1607. 
[13] W.F. Yao, H. Wang, X. H. Xu, S.X. Shang, Y. Hou, Y. Zhang, M. Wang, Synthesis and photocatalytic property of bismuth titanate $\mathrm{Bi}_{4} \mathrm{Ti}_{3} \mathrm{O}_{12}$, Mater. Lett. 57 (2003) 1899-1902.

[14] W.F. Yao, X.H. Xu, H. Wang, J.T. Zhou, X. N. Yang, Y. Zhang, S. X. Shang, B.B. Huang, Photocatalytic property of perovskite bismuth titanate, Appl. Catal. B 52 (2004) 109-116.

[15] L. Xie, J. Ma, H. Tian, J. Zhou, Z. Zhao, P. Wu, Y. Hu, Y. Wang, J. Tao, X. Zhu, Isopropanol-assisted hydrothermal synthesis of bismuth titanate nanophotocatalysts, Mat. Lett. 60 (2006) 284-286.

[16] L. Zheng, G. Yan, Y. Huang, X. Wang, J. Long, L. Li, T. Xu, Visible-light photocatalytic denitrogenation of nitrogen-containing compound in petroleum by metastable $\mathrm{Bi}_{20} \mathrm{TiO}_{32}$, Inter. J. Hydrogen Energy 39 (2014) 13401-13407.

[17] W-N. Su, D.W. Ayele, V. Ochie, C-J. Pan, B-J Hwang, The development of highly crystalline single-phase $\mathrm{Bi}_{20} \mathrm{TiO}_{32}$ nanoparticles for light driven oxygen evolution, Appl. Catal. B 150-151 (2014) 363-369.

[18] H. Cheng, B. Huang, Y. Dai, X. Qin, X. Zhang, Z. Wang, M. Jiang, Visible-light photocatalytic activity of the metastable $\mathrm{Bi}_{20} \mathrm{TiO}_{32}$ synthesized by a hightemperature quenching method, J. Solid State Chem. 182 (2009) 2274-2278.

[19] Y. Hou, M. Wang, X. Xu, H. Wang, S. Shang, D. Wang, W. Yao, $\mathrm{Bi}_{20} \mathrm{TiO}_{32}$ nanocones preprared from Bi-Ti-O mixture by metalorganic decomposition method, J. Crystal Growth 240 (2002) 489-494.

[20] T. Zhou, J. Hu, Mass production and photocatalytic activity of highly crystalline metastable single-phase $\mathrm{Bi}_{20} \mathrm{TiO}_{32}$ nanosheets, Environ. Sci. Technol. 44 (2010) 8698-8703. 
[21] E. Grabowska, J. Reszczyńska, A. Zaleska, Mechanim of phenol photodegradation in the presence of pure and modified- $\mathrm{TiO}_{2}$ : A review, Water Res. 46 (2012) 54535471.

[22] G. Busca, S. Berardinelli, C. Resini, L. Arrighi, Technologies for the removal of phenol from fluid streams: A short review of recent developments, J. Hazardous. Mater. 160 (2008) 265-288.

[23] M. Rochkind, S. Pasternak, Y. Paz, Using Dyes for Evaluating Photocatalytic Properties: A Critical Review, Molecules 20 (2015) 88-110.

[24] S. Murcia-López, M.C. Hidalgo, J.A. Navío, Synthesis, characterization and photocatalytic activity of $\mathrm{Bi}$-doped $\mathrm{TiO}_{2}$ photocatalysts under simulated solar irradiation, Appl. Catal. A 404 (2011) 59-67.

[25] M.C. Hidalgo, G. Colón, J.A. Navío, Modification of the physicochemical properties of commercial $\mathrm{TiO}_{2}$ samples by soft mechanical activation, J. Photochem. Photobiol. A 148 (2002) 341-348.

[26] A.Q. Jiang, H.G. Li, L.D. Zhang, Dielectric study in nanocrystalline $\mathrm{Bi}_{4} \mathrm{Ti}_{3} \mathrm{O}_{12}$ prepared by chemical coprecipitation, J. Appl. Phys. 83 (1998) 4878-4883.

[27] R. He, S. Cao, P. Zhou, J. Yu, Recent advances in visible light Bi-based photocatalysts, Chin. J. Catal. 35 (2014) 989-1007.

[28] X. Zhang, L. Zhang, J-S. Hu, C-L- Pan, C-M. Hou, Facile hydrothermal synthesis of novel $\mathrm{Bi}_{12} \mathrm{TiO}_{20}-\mathrm{Bi}_{2} \mathrm{WO}_{6}$ heterostructure photocatalyst with enhanced photocatalytic activity, Appl. Surf. Sci. 346 (2015) 33-40.

[29] F. Duo, Y. Wang, X. Mao, X. Zhang, Y. Wang, C. Fan, A BiPO $4 / B^{B O C l}$ heterojunction photocatalyst with enhancedelectron-hole separation and excellent photocatalytic performance, Appl. Surf. Sci. 340 (2015) 35-42. 
[30] Y. Deng, L. tang, G. Zeng, H. Dong, M. Yan, J. Wang, W. Hu, J. Wang, Y. Zhou, J. Tang, Enhanced visible light photocatalytic performance of polyaniline modified mesoporous single Crystal TiO2 microsphere, Appl. Surf. Sci. 387 (2016) 882-893. 


\section{Figures Captions}

Figure 1. XRD patterns for $\mathrm{TiO}_{2} \mathrm{P} 25, \mathrm{BTO}-$ Fresh and $\mathrm{BTO}-400$ samples. "*” indicates $\mathrm{Bi}_{4} \mathrm{Ti}_{3} \mathrm{O}_{12}$ diffraction peaks (PDF-012-02-13) and “ $\Theta$ " indicates $\mathrm{Bi}_{20} \mathrm{TiO}_{32}$ peaks (PDF042-02-02).

Figure 2. UV-Vis DR spectra for BTO-400 (blue line), BTO-Fresh (black line) and $\mathrm{TiO}_{2}$ P25 (red line).

Figure 3. Pore size distributions for BTO-Fresh (red line) and BTO-400 (black line) samples.

Figure 4. SEM pictures of BTO-Fresh (A) and BTO-400 (B).

Figure 5. TEM images of BTO-400 sample presenting different areas (a, b and $\mathbf{d}$ ); HRTEM micrographs showing the interplanar spacing of the two phases, $\mathrm{Bi}_{20} \mathrm{TiO}_{32}$ and $\mathrm{Bi}_{4} \mathrm{Ti}_{3} \mathrm{O}_{12}$ (c, e and $\mathbf{f}$ ) and enlargement of the image presented in $\boldsymbol{e}$, the insets correspond to the FFTs of the square marked areas $(\boldsymbol{g})$.

Figure 6. HRTEM micrographs of the BTO-400 sample and the corresponding FFTs of the square marked areas showing three different zone axes of the $\mathrm{Bi}_{4} \mathrm{Ti}_{3} \mathrm{O}_{12}$ phase.

Figure 7. STEM images of BTO-400 sample obtained from different areas and showing the two positions where the EDX analysis was done ( $\mathbf{a}, \mathbf{b}$ and $\mathbf{c})$. TEM image (d) and STEM image of the same region where two positions were analysed by EDX (e) and EDX spectra obtained from the two positions( $\mathbf{f}$ and $\mathbf{g})$.

Figure 8. Phenol photodegradation profiles over the different photocatalysts under UV-Vis illumination (upper figure) and visible illumination (lower figure).

Figure 9. Initial reaction rates for phenol photodegradation over the different photocatalysts under UV-Visible (left) and just Visible illumination (right). 
Figure 10. Initial reaction rates per surface area unit of photocatalyst for phenol photodegradation under UV-Visible (left) and just Visible illumination (right). 
Table 1. Some characterisation results for the different photocatalysts.

\begin{tabular}{|c|c|c|c|c|}
\hline & $\begin{array}{c}\text { Crystalline phases } \\
\text { (XRD) }\end{array}$ & $\begin{array}{c}\mathrm{Bi} / \mathrm{Ti} \text { Content } \\
\text { (molar, XRF) }\end{array}$ & $\mathrm{S}_{\mathrm{BET}}\left(\mathrm{m}^{2} / \mathrm{g}\right)$ & Band Gap (eV) \\
\hline BTO_Fresh & $100 \% \mathrm{Bi}_{4} \mathrm{Ti}_{3} \mathrm{O}_{12}$ & 1.47 & 46 & 2.9 \\
\hline BTO_400 & $\begin{array}{c}23 \% \mathrm{Bi}_{4} \mathrm{Ti}_{3} \mathrm{O}_{12} \\
77 \% \mathrm{Bi}_{20} \mathrm{TiO}_{32}\end{array}$ & 1.47 & 35 & 2.1 \\
\hline TiO2 P25 & $\begin{array}{c}80 \% \mathrm{TiO}_{2} \text { Anatase } \\
20 \% \mathrm{TiO}_{2} \mathrm{Rutile}\end{array}$ & - & 50 & 3.1 \\
\hline
\end{tabular}




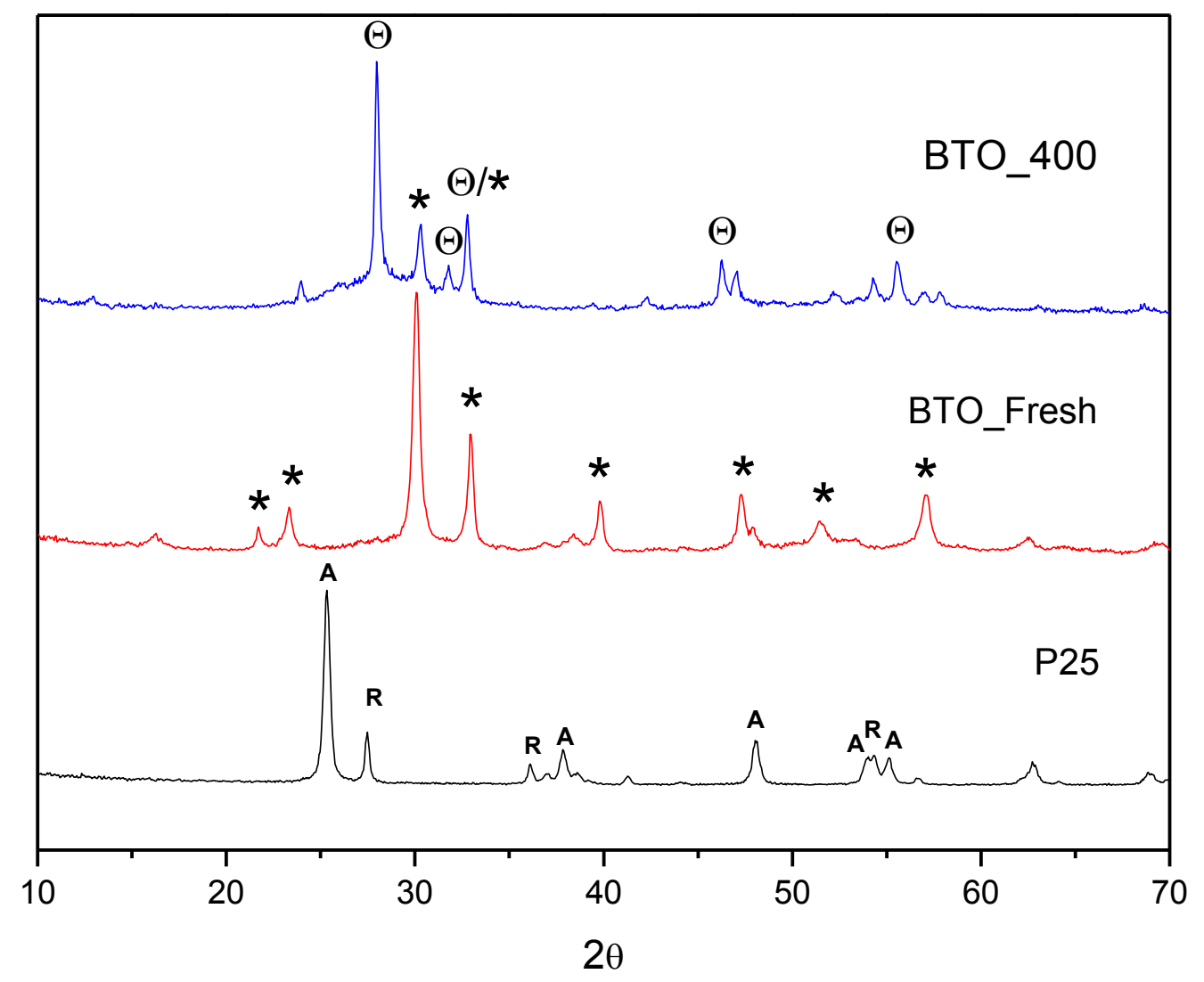




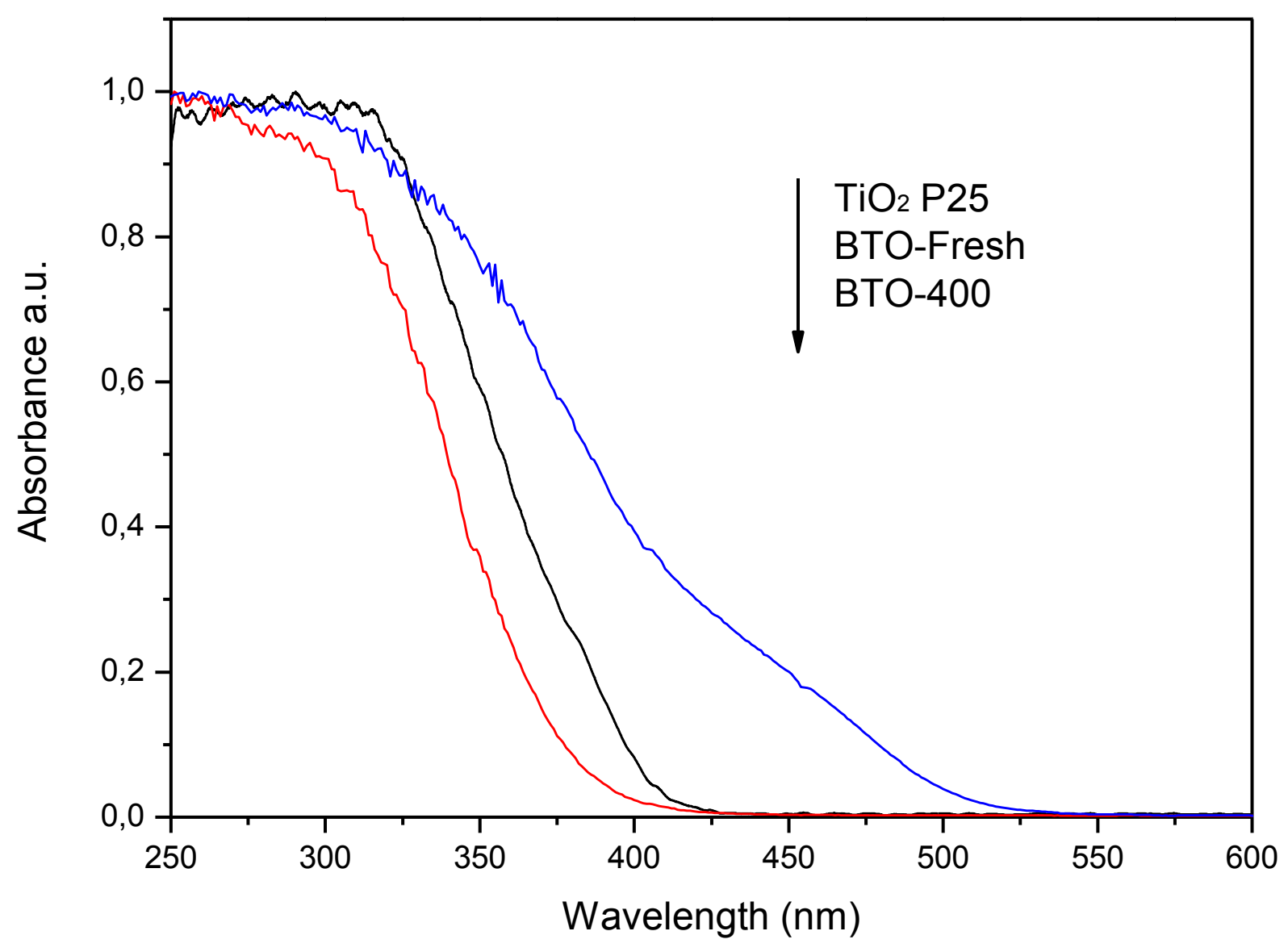




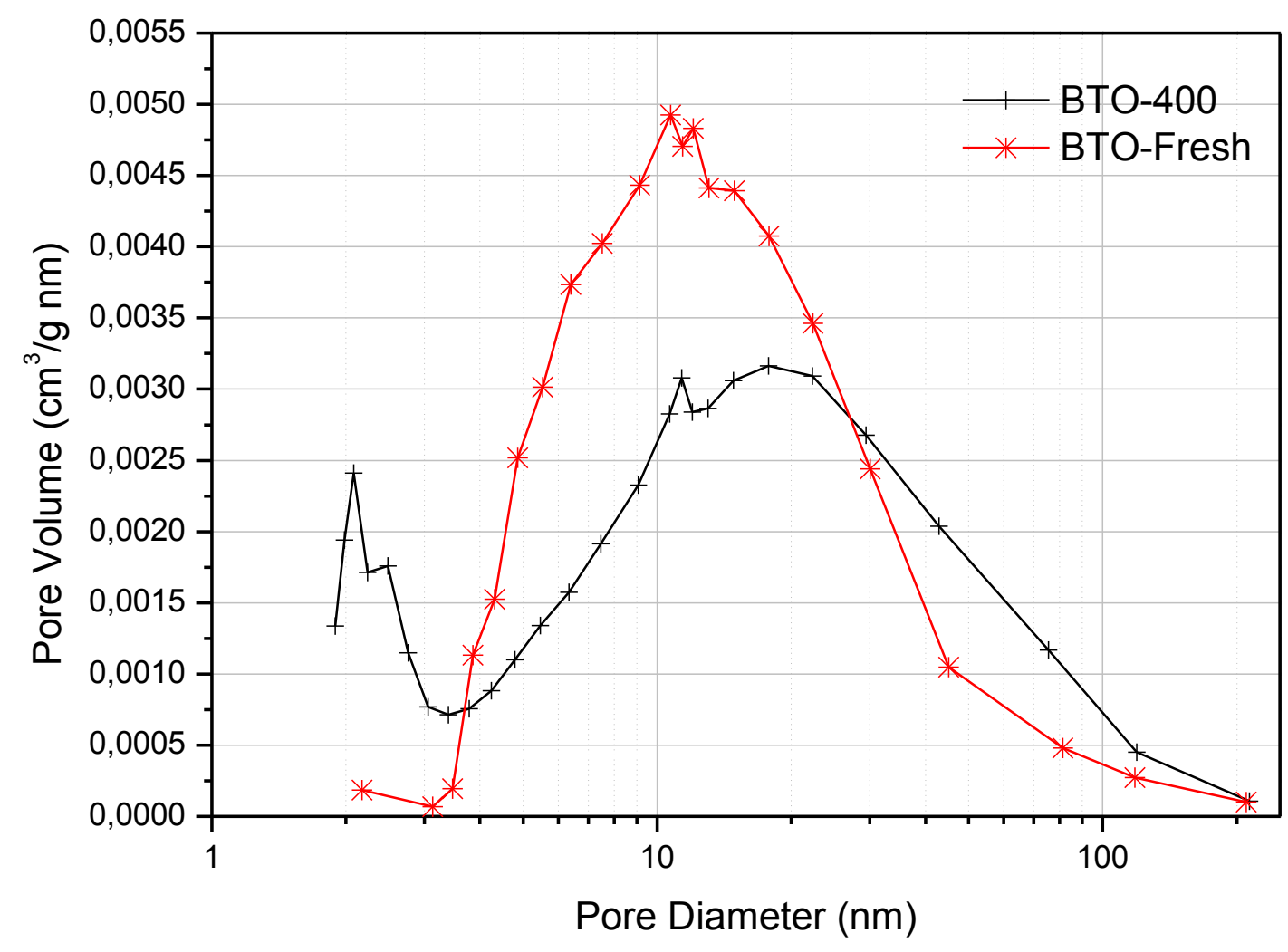


Click here to download Figure: Fig4New.docx

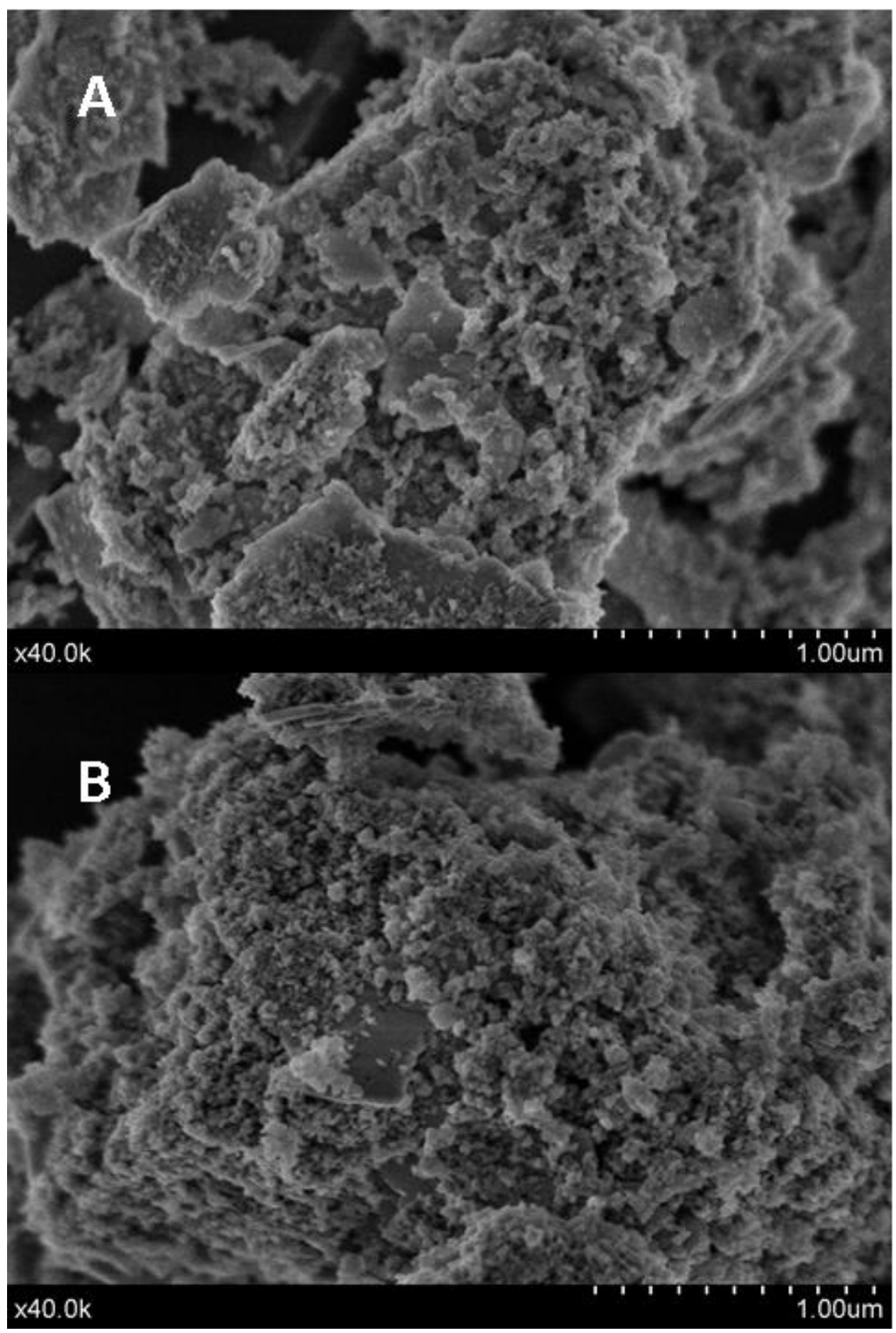


Click here to download Figure: Fig5New.docx
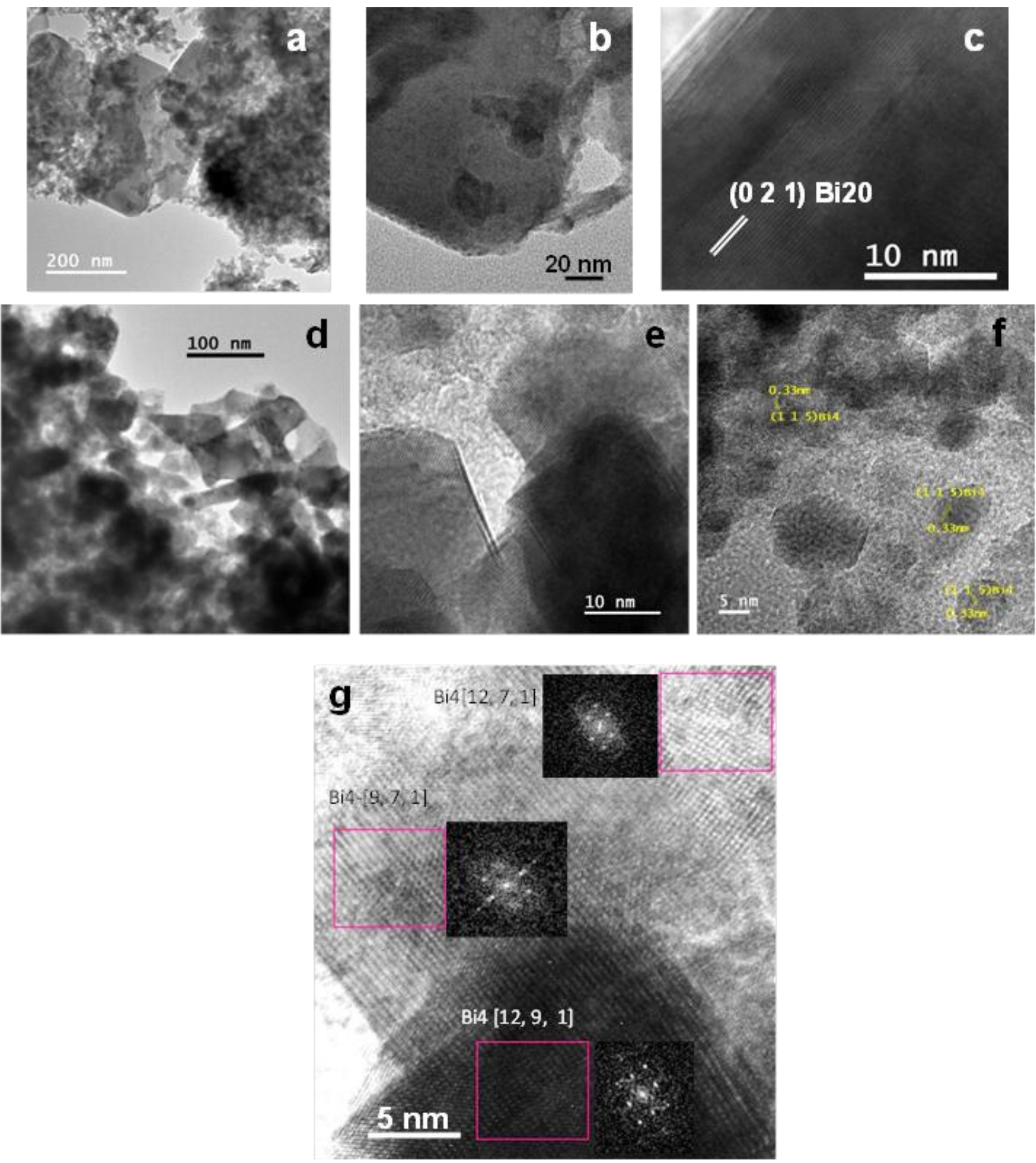
Click here to download Figure: Fig6New.docx
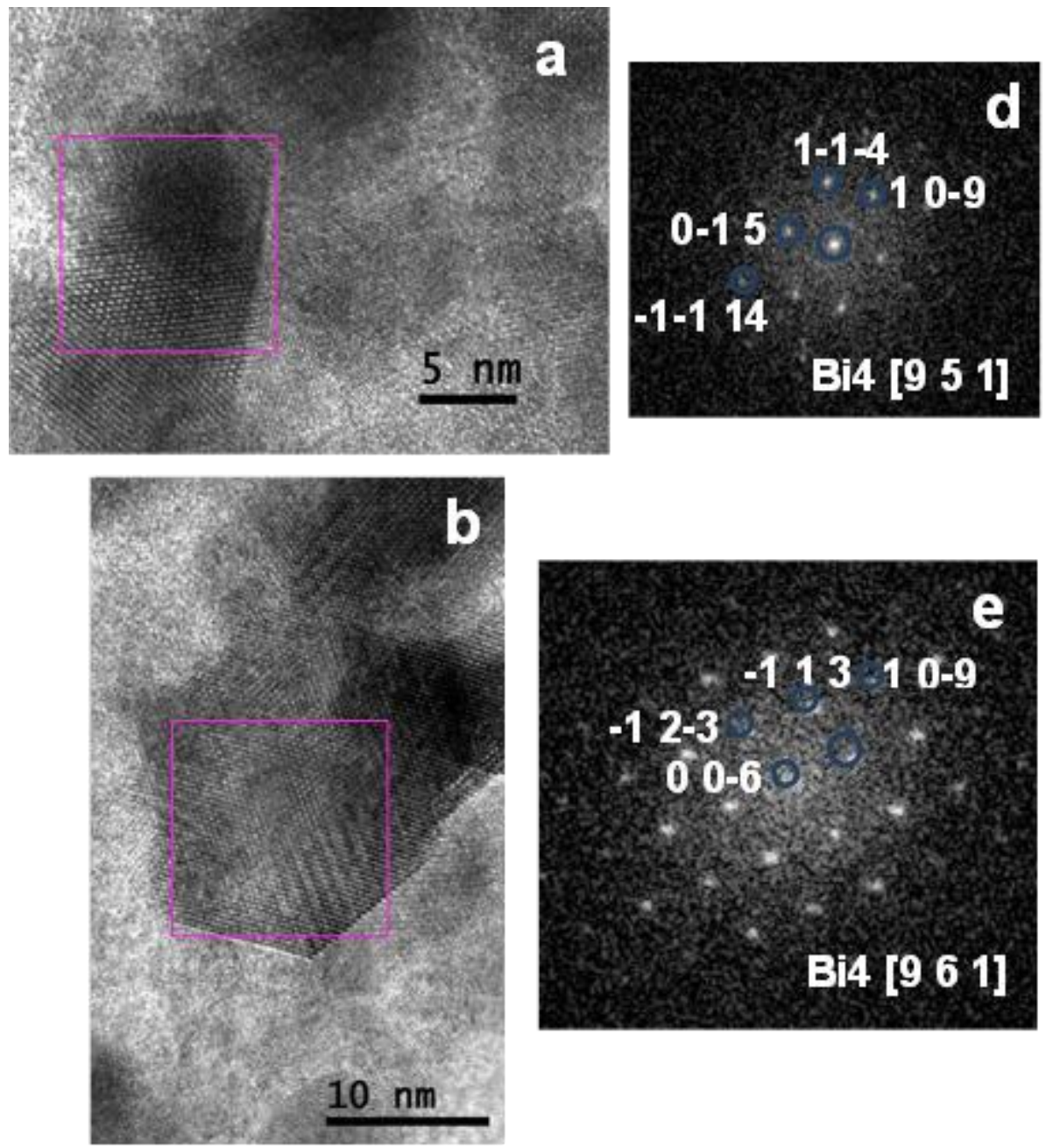

$10 \mathrm{~nm}$
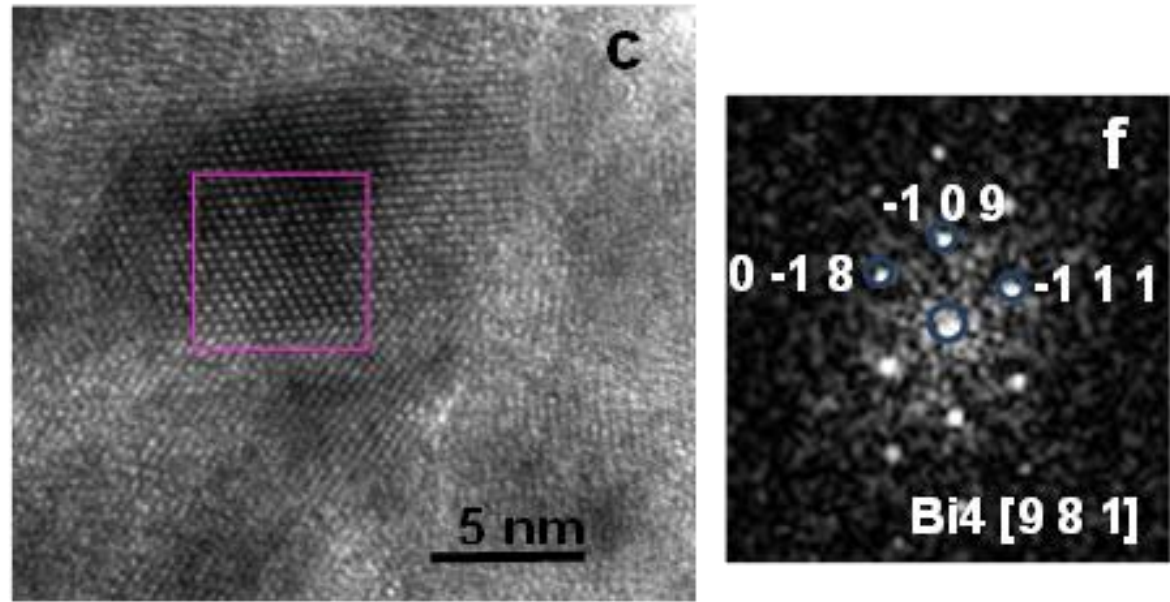


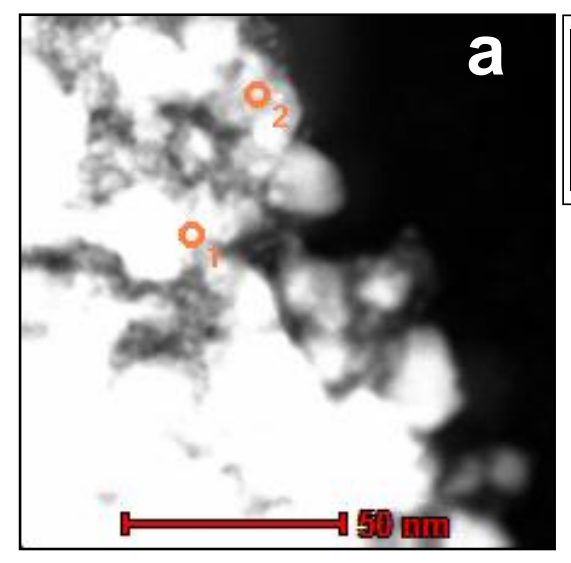

\begin{tabular}{||c|c|c|}
\hline & Bi Atomic \% & Ti Atomic \% \\
\hline 1 & 58 & 42 \\
\hline 2 & 62 & 38 \\
\hline
\end{tabular}

\begin{tabular}{|l|c|c|}
\hline & Bi Atomic \% & Ti Atomic \% \\
\hline 1 & 61 & 39 \\
\hline 2 & 59 & 41 \\
\hline
\end{tabular}

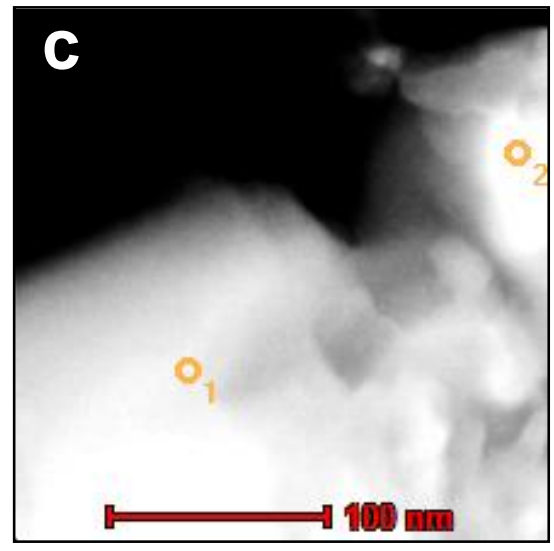

\begin{tabular}{|l|c|c|}
\hline & Bi Atomic \% & Ti Atomic \% \\
\hline 1 & 94 & 6 \\
\hline 2 & 79 & 21 \\
\hline
\end{tabular}
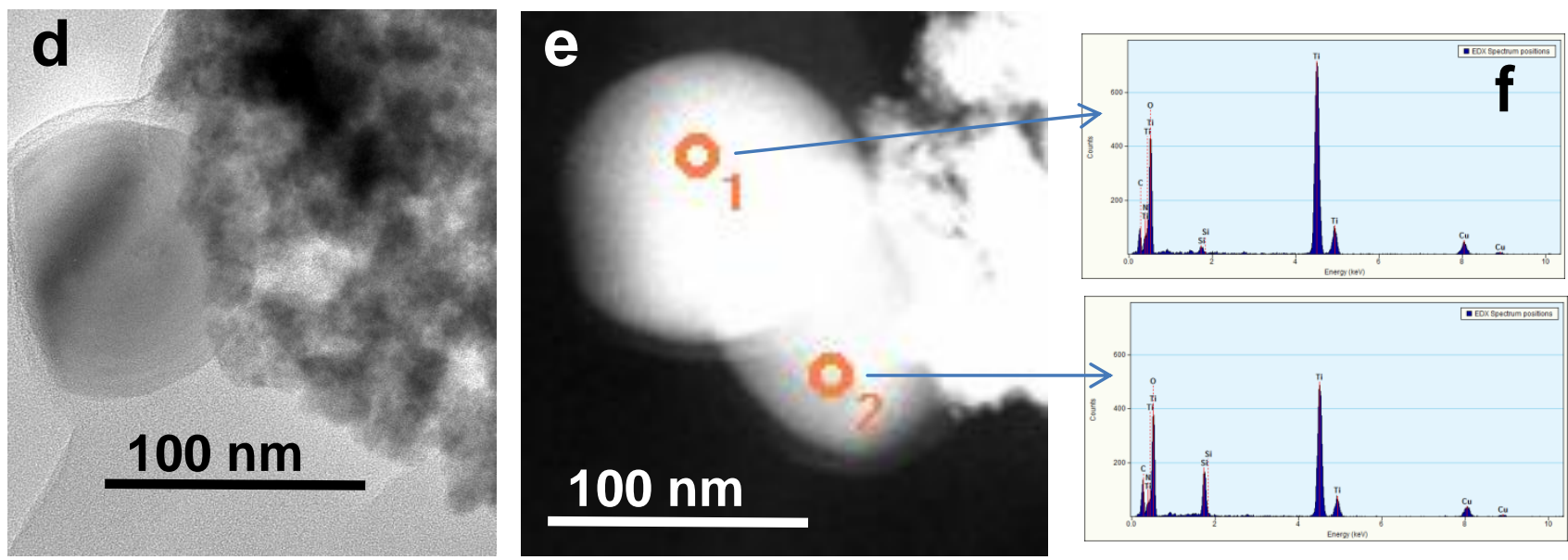

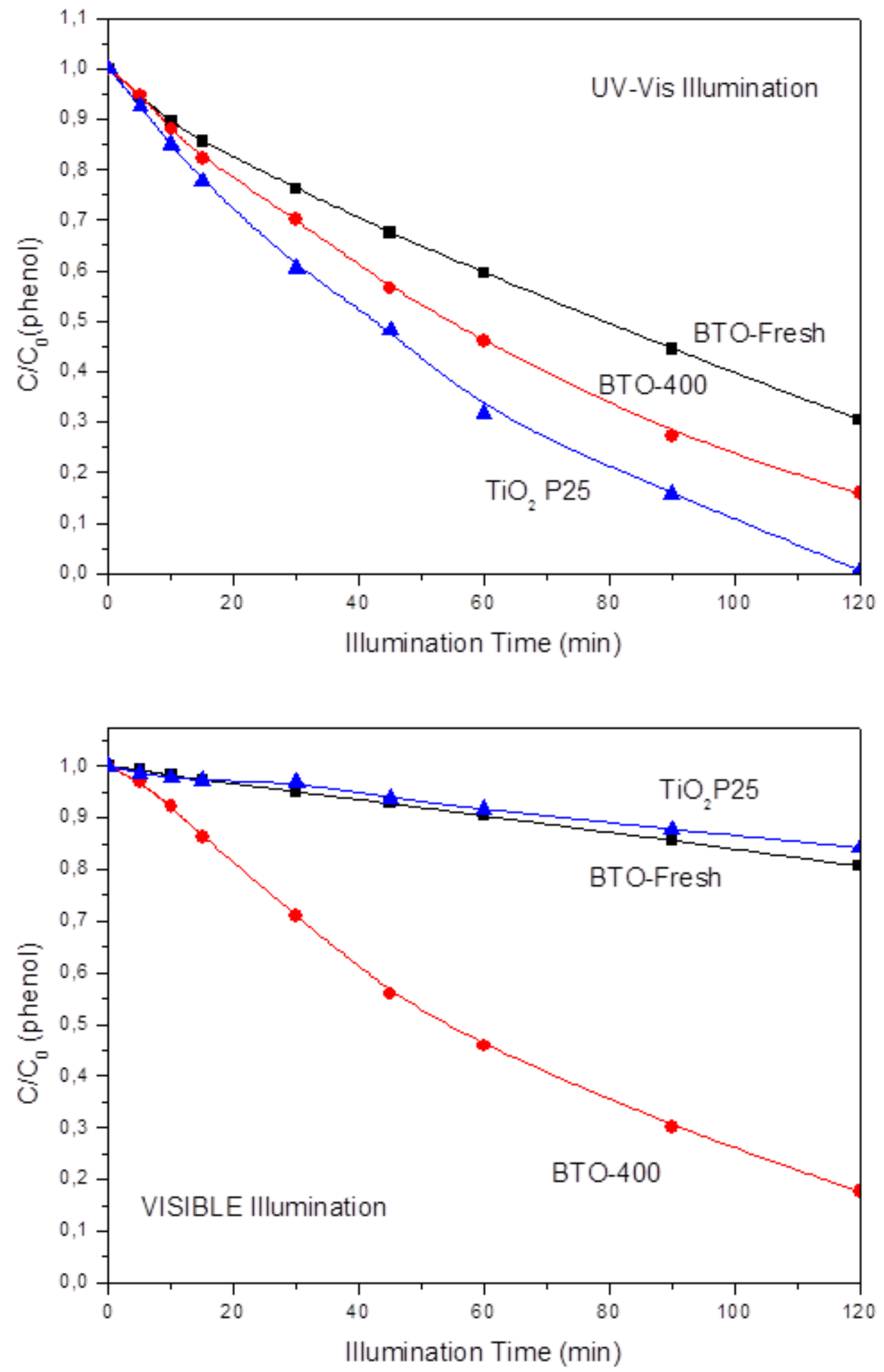


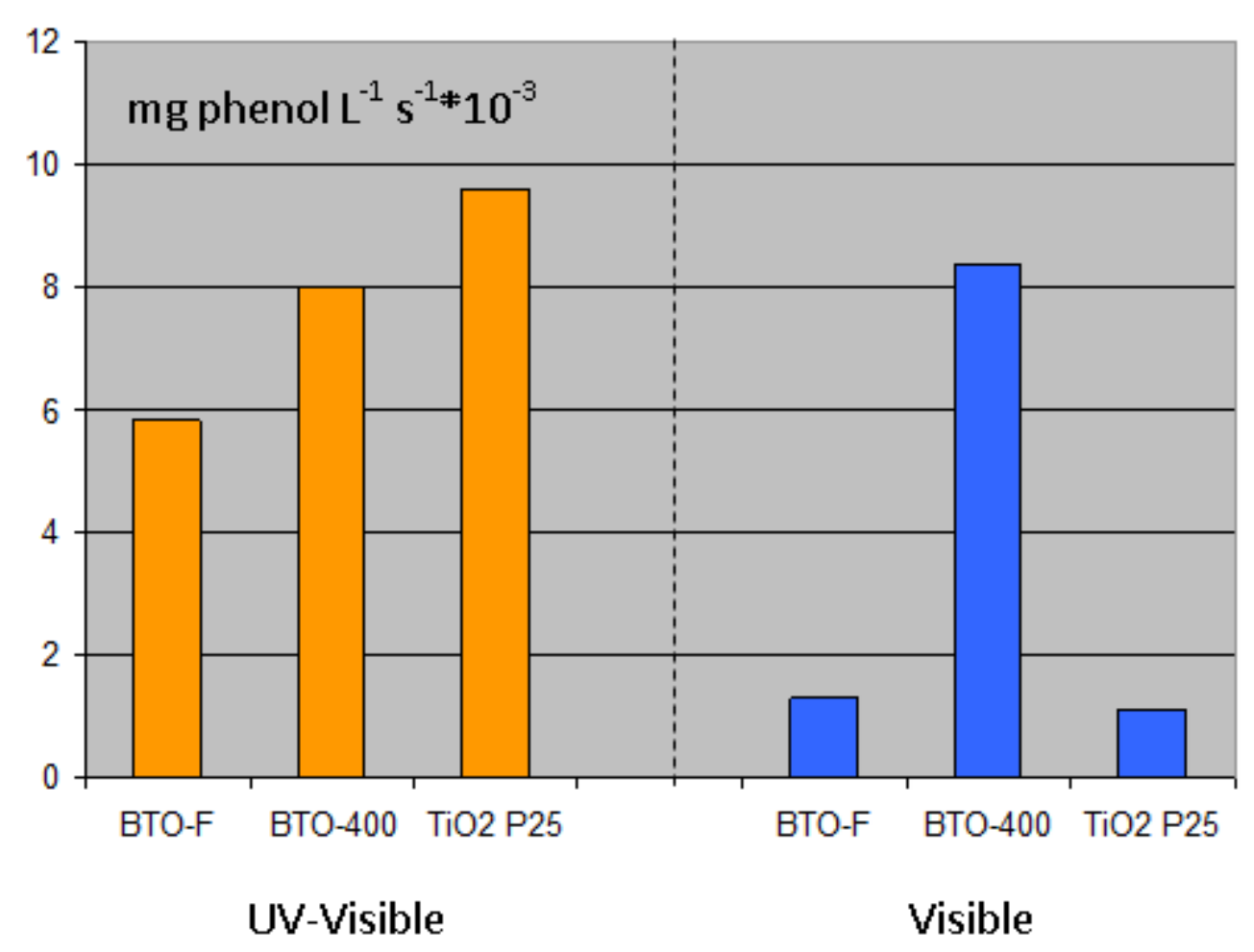




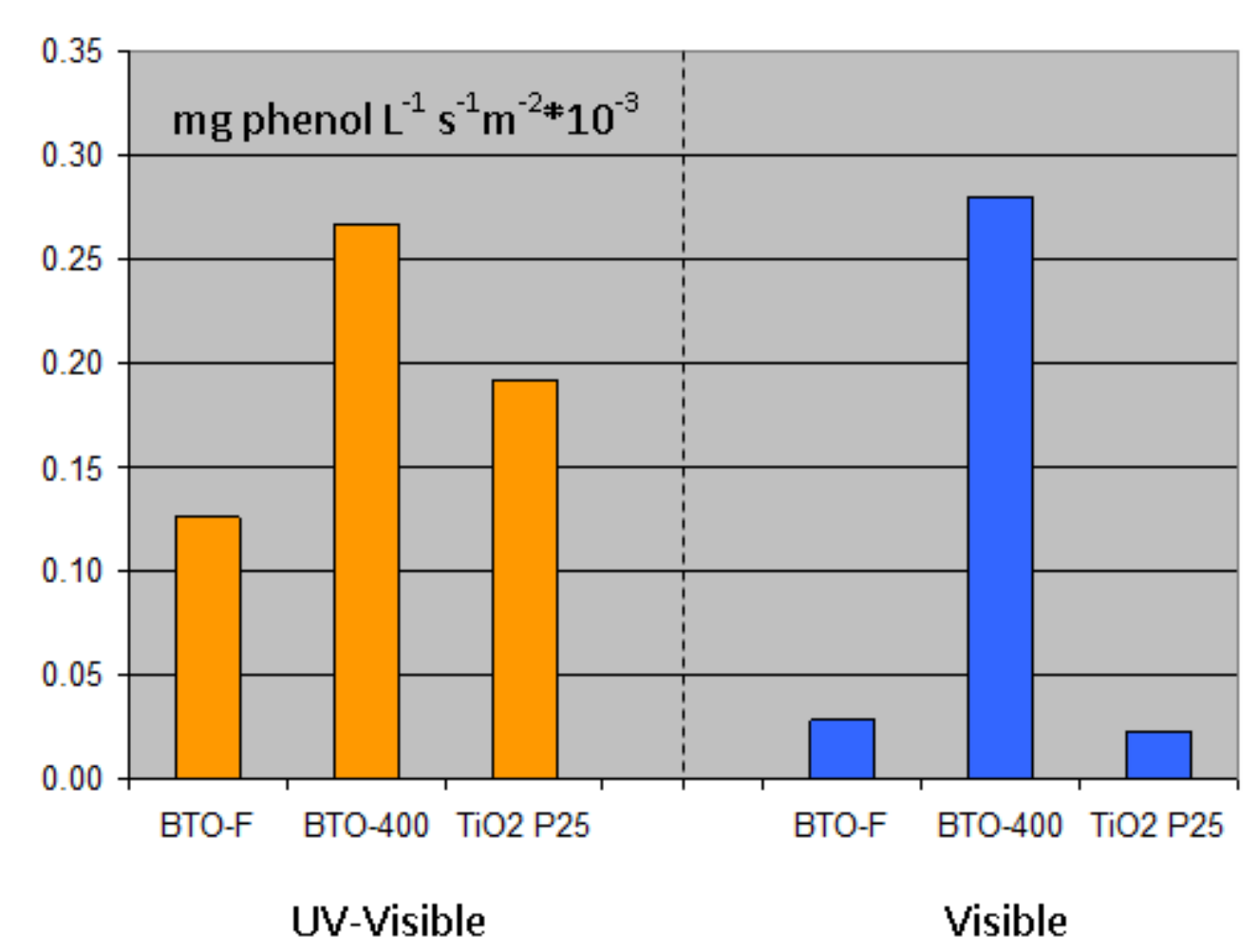

